\title{
Globally optimized cross-correlation for particle image velocimetry
}

\author{
Hongping Wang ${ }^{1}\left(\mathbb{D} \cdot\right.$ Guowei He $^{1,2} \cdot$ Shizhao Wang ${ }^{1,2}$
}

Received: 5 May 2020 / Revised: 25 July 2020 / Accepted: 19 September 2020 / Published online: 10 October 2020

(c) Springer-Verlag GmbH Germany, part of Springer Nature 2020

\begin{abstract}
We propose a global optimization method to automatically search for the correlation peak instead of computing the entire cross-correlation map throughout an interrogation window (IW) using a fast Fourier transform (FFT)-based method. The proposed method, named globally optimized cross-correlation for particle image velocimetry (GOCCPIV), minimizes an objective function consisting of a residual term for cross-correlation and a penalty term for smoothness to solve the optimal velocity field. A very small IW is adopted in GOCCPIV to obtain a dense velocity field with a high spatial resolution. The proposed method is quantitatively validated on synthetic particle image pairs with different flow patterns and is compared with the mainstream FFT-based cross-correlation method (FFTCCPIV) and physical-based optical flow (OpticalFlow). We consider the influences of the IW size, particle concentration, particle image diameter, large displacements and image noise on the velocity measurements. Error analysis indicates that GOCCPIV outperforms FFTCCPIV in resolving small-scale vortices and reducing the measurement error. Finally, the proposed method is applied to a real PIV experiment with an impinging jet. The results indicate that GOCCPIV is more suitable than FFTCCPIV for resolving high-velocity-gradient regions.
\end{abstract}

\section{Graphic abstract}

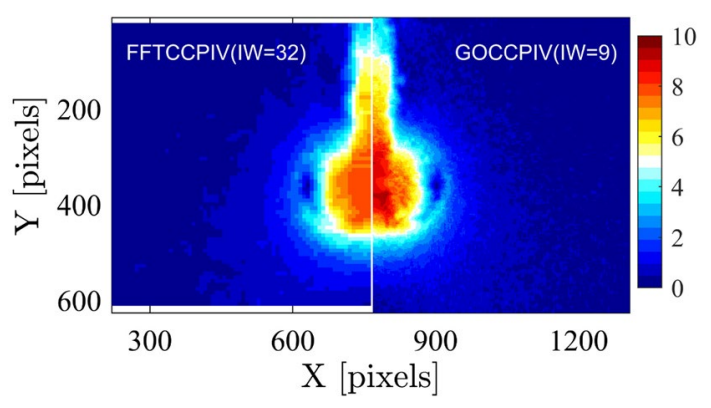

\section{Introduction}

The particle image velocimetry (PIV) technique (Adrian 1991; Willert and Gharib 1991) is widely used to estimate flow motions in the field of experimental fluid mechanics. The greatest advantage of PIV is that it can simultaneously measure many points in a planar or volumetric domain. In

Shizhao Wang

wangsz@lnm.imech.ac.cn

1 The State Key Laboratory of Nonlinear Mechanics, Institute of Mechanics, Chinese Academy of Sciences, Beijing 100190, China

2 School of Engineering Sciences, University of the Chinese Academy of Sciences, Beijing 100049, China a planar PIV experiment, the tracer particles seeded in the flow field are illuminated by a sheet of laser light. Particle images are then recorded at two consecutive instants using a CCD or CMOS camera. Image analysis methods, such as region-based cross-correlation (Huang and Fiedler 1993; Scarano 2002), particle tracking (Adamczyk and Rimai 1988; Maas et al. 1993) or optical flow (Horn and Schunck 1981; Barron et al. 1994; Corpetti et al. 2002), are adopted to estimate the velocity field from the image sequence. Recently, neural networks have also been used to estimate flow motions by learning from acquired datasets (Lee et al. 2017; Cai et al. 2019).

Correlation-based methods are a simple and robust means of assessing displacement by finding the maximum 
of the cross-correlation map within a given interrogation window (IW). The velocity obtained in this way is approximately the average value of the velocity field over the IW, which can be simply modeled as a moving average (Nogueira et al. 1999). To correct for the effects of out-ofplane motion, image noise and low particle concentrations, data validation and smoothing methods are always applied following correlation-based methods (Adrian 2005; Garcia 2011; Wang et al. 2015). For a correlation-based method, choosing an appropriate size for the IW is crucial. If the IW is too small, the resulting field will be contaminated by many outliers and random noise caused by unreliable correlation peaks. If the IW is too large, the resulting field will be oversmoothed by the modulation effect of the IW. This implies that the spatial resolution cannot be improved simply by reducing the size of the IW. To overcome this limitation, the window deformation iterative multigrid (WIDIM) technique (Scarano 2002) has been developed for flow motion estimation. Image deformation techniques can improve the accuracy of velocimetry; however, the spatial resolution is still limited by the IW size because the amplitude response is negative when the size of the IW is larger than the local wavelength of the velocity (Nogueira et al. 1999). To obtain a "super-resolution" flow field, an approach based on second-order velocity gradients has been proposed by Scarano (2004). Susset et al. (2006) used an iterative process to resize the IW in accordance with vector validation, followed by particle tracking velocimetry (PTV) to further determine the displacement of each particle. Data assimilation, which combines the physical models and measured data can also be used to enhance the resolution of velocity fields and passive scalar images (Schneiders and Scarano 2016; Zille et al. 2016). Instead of finding the maximum of the correlation map as performed in correlation-based techniques, the FOLKI-PIV method proposed by Champagnat et al. (2011) uses an iterative gradient-based cross-correlation optimization approach to obtain a dense velocity field (one vector per pixel). This approach has been shown to be as accurate as state-of-the-art standard PIV software while being 50 times faster due to GPU implementation. However, the spatial resolution is almost the same as that of standard PIV due to the same IW.

Optical flow methods are another way to extract flow motion by minimizing a local or global cost function. Generally, this objective function consists of two terms: one is a data modeling term deduced from an assumption of brightness or gradient conservation (Drulea and Nedevschi 2013), and the other is a regularization term used to control the degree of smoothness of the velocity field. Taking the brightness conservation as an example, it is mathematically expressed by a first-order Taylor expansion as follows: $\frac{\partial \mathrm{I}}{\partial t}+\mathbf{U} \cdot \nabla \mathrm{I}=0$,

where $\mathbf{U}=(u, v)$ represents the two-component velocity field in the image plane, $\partial \mathrm{I} / \partial t$ is the partial time derivative of the image intensity $\mathrm{I}$, and $\nabla$ represents the spatial gradient operator. The assumption of brightness conservation seems inappropriate under the conditions of many real PIV experiments. Even for the cross-correlation method, the achievable accuracy is strongly limited by the individual intensity variations of particle images caused by out-ofplane motion (Nobach 2011). To overcome this weakness, a direct approach to optical flow estimation with zero-mean normalized cross-correlation (ZNCC) was proposed by Drulea and Nedevschi (2013). This approach was shown to be very robust to illumination changes. Moreover, Liu and Shen (2008) explored the relationship between fluid flow and optical flow and provided a physically based data term for image-based fluid velocity measurements. Cassisa et al. (2011) used a scalar transport equation to define the flow equation and modeled the small scales using a subgrid-scale model. Because a data term such as Eq. 1 is ill posed, a regularization term must be incorporated to solve for the velocity field $\mathbf{U}$. This term can be reconstructed using a local approach such as the Lucas-Kanade method (Lucas and Kanade 1981) or a global approach such as the HornSchunck method (Horn and Schunck 1981) and the div-curl smoothing term (Corpetti et al. 2006; Zhong et al. 2017). With these regularization terms, the dense velocity field can be iteratively solved in the frame of optical flow. Further discussions can be found in the papers by Zhong et al. (2017) and Seong et al. (2019).

Between correlation-based and optical flow methods, the former represent a kind of intensity integration approach to finding the displacement, while the latter represents an intensity differential approach, as shown in Eq. 1. Currently, correlation-based methods are more commonly used than optical flow methods for PIV. Additionally, the smoothness of a correlation-based method is determined by the size of the IW instead of by the regularization term, as in optical flow methods. To refine the measured velocity fields, efforts have been made to combine the advantages of both types of methods. Heitz et al. (2008) defined a new energy function to constrain the estimated displacements to be close to a sparse-correlation-based vector field. Becker et al. (2012) proposed a variational adaptive correlation method to jointly optimize the displacement field and the shape of a Gaussian IW; the displacement field is solved by maximizing the correlation object function, and the IW shapes are steered by the error model function. Seong et al. (2019) proposed a method to enhance the measured resolution by using the global optical flow along with the image deformation deduced via correlation-based PIV. 
For a correlation-based PIV method, the spatial resolution is limited by the number of particles in the IW. A potential way to reduce the size of the IW is to use the variational method of optical flow to constrain the velocity field. Therefore, in this study, a global optimization method with a regularization term is used to directly find the maximum of the cross-correlation map. This approach, named globally optimized cross-correlation particle image velocimetry (GOCCPIV), uses a gradient-based optimization algorithm to minimize an objective function consisting of a crosscorrelation term and a smoothness term. The GOCCPIV method not only avoids the assumption of brightness conservation but also improves the spatial resolution by enabling the adoption of a very small IW. Notably, this idea of combining cross-correlation and global optimization is similar to the combined local-global optical flow method proposed by Bruhn et al. (2005), where the local Lucas-Kanade method is integrated into the global Horn-Schunck technique. This approach is used in the field of computer vision. The rest of this paper is organized as follows. In Sect. 2, we first present our globally optimized cross-correlation approach, which is efficiently computed using a state-of-the-art optimization algorithm and integrated with image deformation. In Sect. 3, the proposed method is compared with the correlation-based PIV algorithm and physics-based optical flow approach on synthetic images. In Sect. 4, some real experimental images are used to evaluate this new method. Finally, we conclude in Sect. 5.

\section{Methodology}

\subsection{Globally optimized cross-correlation}

In the original PIV algorithm, the displacement is obtained by searching for the maximum peak in the cross-correlation map and then applying subpixel interpolation to estimate the precise displacement. This procedure is actually an optimization process for seeking a displacement by maximizing the intensity match. As proposed by Champagnat et al. (2011), cross-correlation maximization can be achieved by means of a gradient-based optimization method instead of by calculating the cross-correlation scores of all possible displacements, as in the original PIV algorithm. The cross-correlation is replaced by the sum of the squared differences (SSD) in the IW, and the Gauss-Newton iteration is derived in accordance with the first-order Taylor expansion. A detailed explanation of the relationship between the cross-correlation and SSD can be found in the paper by Drulea and Nedevschi (2013). In contrast to the method proposed by Champagnat et al. (2011), we still compute the cross-correlation as defined in the original correlationbased methods; however, a regularization term is added to the correlation-based algorithm to overcome the problems caused by a small IW. This can be achieved by optimizing the following formula:

$R(\mathbf{u}, \mathbf{v})=\int_{\Omega}(\underbrace{\left(1-\mathbf{C}_{\mathbf{u}, \mathbf{v}}\right)^{2}}_{\text {residual of correlation }}+\underbrace{\gamma_{1}^{2}(\Delta \mathbf{u})^{2}+\gamma_{2}^{2}(\Delta \mathbf{v})^{2}}_{\text {smoothness of the field }}) d \mathbf{s}$,

where $\mathbf{U}=(\mathbf{u}, \mathbf{v})$ represents the displacement field, $\mathbf{C}_{\mathbf{u}, \mathbf{v}}$ is the correlation map corresponding to the displacement field, and the parameters $\gamma_{1}$ and $\gamma_{2}$ are real positive scalars that control the degree of smoothness. The objective function is minimized over the whole measurement domain $\Omega$. Given the displacement field, the normalized cross-correlation coefficient is calculated over the IW at each grid node as follows:

$\mathbf{C}_{\mathbf{u}, \mathbf{v}}=\frac{\sum_{i=1}^{m} \sum_{j=1}^{n} f^{\prime}(i, j) g^{\prime}(i+\mathbf{u}, j+\mathbf{v})}{\sqrt{\sum_{i=1}^{m} \sum_{j=1}^{n} f^{\prime 2}(i, j) \sum_{i=1}^{m} \sum_{j=1}^{n} g^{\prime 2}(i, j)}}$,

where the size of the IW is $m \times n$. The parameters $f^{\prime}$ and $g^{\prime}$ are the gray intensity fluctuations obtained by subtracting the mean value over the IW. The value range of $\mathbf{C}$ is between -1 and 1 . Mean value subtraction can eliminate the contribution of the mean intensity to the cross-correlation (Scarano 2002; Raffel et al. 2018; Scharnowski and Kähler 2020). Figure 1 presents a schematic diagram of the cross-correlation computation at one location. A and B represent the IW in the image pair, and the $C_{u, v}$ at this location can be directly computed by substituting A and B into Eq. 3. The terms on the right-hand side of Eq. 2 consist of a correlation residual term and a penalty term that controls the smoothness of the velocity field. This global smoothness term can constrain the neighboring vectors when the IW is small. In this work, the second-order divided difference form proposed by Garcia (2010) is adopted; this is denoted by the Laplace operator

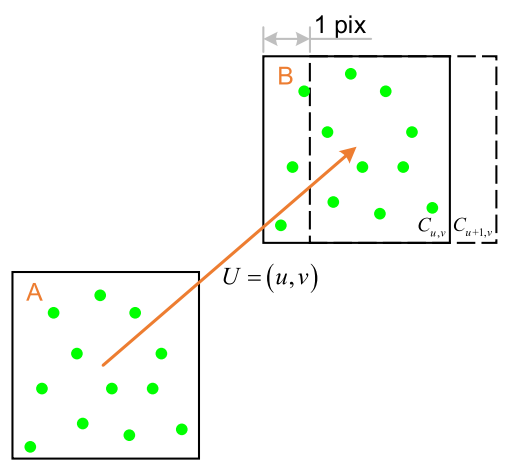

Fig. 1 Schematic diagram of the computation of $C_{u, v}$ and $C_{u+1, v}$ at one location 
$\Delta$ in Eq. 2. Another form of smoothness term used in optical flow can also be adopted in the proposed method, such as Horn-Schunck regularization (Horn and Schunck 1981) or a div-curl constraint term (Corpetti et al. 2006; Zhong et al. 2017). To construct a linear system, the velocity field is reshaped as a column vector, and the Laplace operator is replaced with the difference matrix $D_{x x}+D_{y y}$, where $D_{x x}$ and $D_{y y}$ are the square second-order difference matrices along the $x$ and $y$ directions, respectively. Repeated border elements are adopted to address the boundary conditions (Garcia 2010).

\subsection{Gradient-based minimization}

To solve for the velocity field, we must minimize the objective function $R(\mathbf{u}, \mathbf{v})$. Many state-of-the-art algorithms can be used to solve this nonlinear least-squares problem. In this work, we use the Levenberg-Marquardt method (Levenberg 1944; Marquardt 1963), which is a damped Gauss-Newton method, to solve this problem. The residual array $\mathbf{f}$ is written as follows:

$\mathbf{f}=\left(\begin{array}{c}1-\mathbf{C}_{\mathbf{u}, \mathbf{v}} \\ \gamma_{1} \Delta \mathbf{u} \\ \gamma_{2} \Delta \mathbf{v}\end{array}\right)$

where $\mathbf{f}$ is a vector with dimensions of $3 N \times 1$, and $\mathrm{N}$ is the total number of vectors. To reduce the computation time, the Jacobian array $\mathbf{J}$ of the residuals is also given as follows:

$\mathbf{J}=\frac{\partial \mathbf{f}}{\partial \mathbf{U}}=\left(\begin{array}{cc}-\frac{\partial \mathbf{C}_{\mathbf{u}, \mathbf{v}}}{\partial \mathbf{u}} & -\frac{\partial \mathbf{C}_{\mathbf{u}, \mathbf{v}}}{\partial \mathbf{v}} \\ \gamma_{1}\left(D_{x x}+D_{y y}\right) & \mathbf{0} \\ \mathbf{0} & \gamma_{2}\left(D_{x x}+D_{y y}\right)\end{array}\right)$.

The size of $\mathbf{J}$ is equal to $3 N \times 2 N$. The terms $-\partial \mathbf{C}_{\mathbf{u}, \mathbf{v}} / \partial \mathbf{u}$ and $-\partial \mathbf{C}_{\mathbf{u}, \mathbf{v}} / \partial \mathbf{v}$ represent the gradients of the correlation coefficients with respect to the displacement. To compute the Jacobian array with high efficiency, the displacement field $\mathbf{U}$ is rounded to integer values, and a finite central difference calculation with a step size of \pm 1 pixel is adopted to estimate the gradients $\partial \mathbf{C}_{\mathbf{u}, \mathbf{v}} / \partial \mathbf{u}$ and $\partial \mathbf{C}_{\mathbf{u}, \mathbf{v}} / \partial \mathbf{v}$, as shown in Fig. 1. Taking $\partial \mathbf{C}_{\mathbf{u}, \mathbf{v}} / \partial \mathbf{u}$ as an example, $\mathbf{C}_{\mathbf{u}+\mathbf{1 , v}}$ and $\mathbf{C}_{\mathbf{u}-\mathbf{1 , v}}$ are calculated by shifting the IW by \pm 1 pixel in the $x$ direction around the point $(\mathbf{u}, \mathbf{v})$; then, $\partial \mathbf{C}_{\mathbf{u}, \mathbf{v}} / \partial \mathbf{u}$ is estimated as $\left(\mathbf{C}_{\mathbf{u}+\mathbf{1}, \mathbf{v}}-\mathbf{C}_{\mathbf{u}-\mathbf{1 , v}}\right) / 2$. To reduce the computation time for cross-correlation, the calculated correlation coefficients are stored in memory and will not be repeatedly computed. Note that the arrays $\partial \mathbf{C}_{\mathbf{u}, \mathbf{v}} / \partial \mathbf{u}$ and $\partial \mathbf{C}_{\mathbf{u}, \mathbf{v}} / \partial \mathbf{v}$ are diagonal matrices because the correlation coefficients are assumed to be independent of the adjacent vectors.

The Jacobian array $\mathbf{J}$ and the residual array $\mathbf{f}$ can be computed from the initial displacement field $\mathbf{U}_{0}$. The displacement field $\mathbf{U}$ can then be iteratively updated by solving the following equations:

$$
\begin{aligned}
\left(\mathbf{J}^{\mathbf{T}} \mathbf{J}+\mu \mathbf{I}\right) \mathbf{U}_{l m} & =-\mathbf{J}^{\mathbf{T}} \mathbf{f}, \\
\mathbf{U} & =\mathbf{U}_{0}+\mathbf{U}_{l m},
\end{aligned}
$$

where the damping parameter $\mu(\mu>0)$ influences both the direction and size of the velocity step $\mathbf{U}_{l m}$ and is controlled by the Levenberg-Marquardt method itself. This system of equations is solved using the least-squares $\mathrm{QR}$ factorization method. This process can be repeated many times until the convergence condition is satisfied. Only 10 iterations are adopted in the present globally optimized cross-correlation method. Note that in this type of optimization, if the initial displacement is far from the real solution, there is a risk of obtaining results that are even farther away through further iteration. Figure 2 illustrates an example of a crosscorrelation function with a IW size of $25 \times 25$ pixels, which contains a distinct highest peak corresponding to the particle displacement and many lower noise peaks due to the image noise, velocity gradient and out-of-plane motion. Because the optimization performance is determined by the local gradient of cross-correlation coefficients $\left(\partial \mathbf{C}_{\mathbf{u}, \mathbf{v}} / \partial \mathbf{u}\right.$ and $\left.\partial \mathbf{C}_{\mathbf{u}, \mathbf{v}} / \partial \mathbf{v}\right)$, the optimization will converge to the local noise peak with a wrong initialization. As illustrated in Champagnat et al. (2011), however, when the search is initialized at a value that is not too far (e.g., 2-3 pixels) from the true displacement, such an optimization method can obtain an accurate result within only a few iterations.

\subsection{Parameter optimization}

The parameters $\gamma_{1}$ and $\gamma_{2}$ are positive scalar weights that control the degrees of smoothness of $\mathbf{u}$ and $\mathbf{v}$, respectively. With proper smoothing parameters, over- and undersmoothing can be avoided as much as possible. For fluid motion

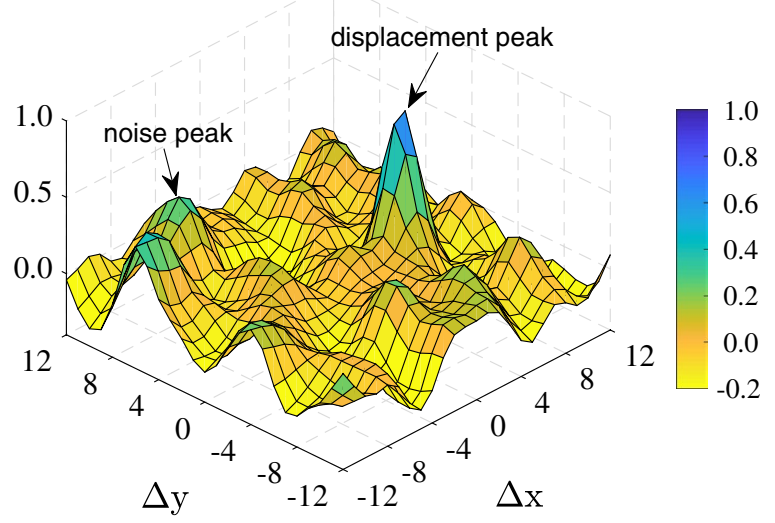

Fig. 2 Example of cross-correlation map with a IW size of $25 \times 25$ pixels 
estimation, the use of $\gamma_{1}$ and $\gamma_{2}$ values that are invariant over different types of flow fields or even different snapshots of the same flow field is impractical. To match the range of residuals in Eq. 4, the residual terms $\Delta \mathbf{u}$ and $\Delta \mathbf{v}$ are normalized with respect to their absolute maxima. This means that the parameters $\gamma_{1}$ and $\gamma_{2}$ can be expressed as follows:

$\gamma_{1}=\frac{\alpha}{\max (|\Delta \mathbf{u}|)}, \gamma_{2}=\frac{\alpha}{\max (|\Delta \mathbf{v}|)}$,

where the value of $\alpha$ is between 0 and 1 . The effect of $\alpha$ on the error is evaluated with the one-dimensional sinusoidal shear displacement, which is introduced in detail in Sect. 3.2, and the results are presented in Fig. 3. This figure shows that the error first decreases and then increases as $\alpha$ increases. In this case, the optimal $\alpha$ values for IW $=5$, 11,17 , and 23 pixels are approximately $0.9,0.5,0.4$, and 0.2 , respectively, and decrease with increasing IW size. This implies that the value of $\alpha$ should be inversely proportional to the IW size due to the smoothing effect of the IW. In this work, an empirical value of $\alpha$ is linearly calculated as follows:

$\alpha=\left\{\begin{array}{rr}1, & I W \leq 5 \\ \frac{I W-32}{5-32}, & 5<I W<32 \\ 0, & I W \geq 32 .\end{array}\right.$

If the IW is larger than 32 pixels (a size that is commonly used in the PIV community), then the GOCCPIV algorithm will be equivalent to the original PIV algorithm. The IW size is related to the particle concentration. If the particle

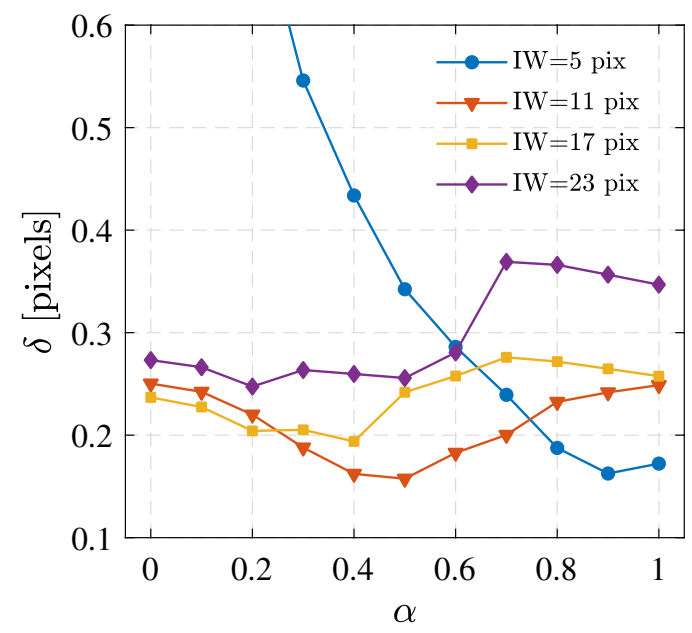

Fig. 3 Error as a function of $\alpha$ for different IW sizes. The y-axis is the error simulated based on the one-dimensional sinusoidal shear displacement, which is introduced in detail in Sect. 3.2. No image pyramids are adopted concentration is very high and an IW with a size of 16 pixels is reliable for estimating the displacements, then the value of $\alpha$ can be set to 0 at IW $=16$ pixels. As the IW decreases in size, the smoothing parameter $\alpha$ gradually increases to 1 to suppress the noise caused by a small IW. This simple configuration can yield a more robust and accurate estimation of the velocity field, as shown in the next section.

Notably, the velocity field obtained by solving Eq. 6 usually is not accurate at the subpixel level because the gradients of the correlation coefficients are calculated based on rounded pixels. Therefore, interpolation of the subpixel displacements by performing Gaussian fitting on the correlation map $\mathbf{C}_{\mathbf{u}, \mathbf{v}}$ is necessary.

\subsection{Overall algorithm and general comments}

The overall GOCCPIV algorithm is summarized in Table 1 . The step size defines the space between adjacent vectors. If the step size is 1 , then all vectors at each pixel are estimated. GOCCPIV not only is a gradient-based optimization algorithm for finding the maximum of the correlation map but also can constrain the vectors to a smooth field by means of a penalty term that reflects the roughness of the data. The greatest benefit provided by the penalty term is that the IW can be set to a much smaller size than is possible in the original PIV algorithm. For GOCCPIV, the optimal IW size is between 5 pixels and 15 pixels, depending on the particle concentration and image noise. Before setting the parameters, the computational efficiency of GOCCPIV, which is determined by the IW size and step size, must be considered. A larger IW will require more time to compute the normalized cross-correlation coefficients. Therefore, the computation time increases with increasing IW size. The step size determines the number of vectors. If the step size is large, Eq. 6 can be quickly solved due to the low array dimensions.

Table 1 Pseudocode for GOCCPIV

\begin{tabular}{|c|c|}
\hline 1 & Input: Image A and Image B \\
\hline 2 & Output: $\mathbf{U}=(\mathbf{u}, \mathbf{v})$ \\
\hline 3 & Begin \\
\hline 4 & $\begin{array}{l}\text { Set the parameters: IW size, step size, and number of opti- } \\
\text { mization iterations }\end{array}$ \\
\hline 5 & $\begin{array}{l}\text { Calculate the smoothing parameter } \alpha \text { in accordance with Eq. } \\
8\end{array}$ \\
\hline 6 & Generate the location mesh for the vectors: xmesh,ymesh \\
\hline 7 & Generate the Laplace operator: $D_{x x}+D_{y y}$ \\
\hline 8 & Initialize the velocity field $\mathbf{U}$ as $\mathbf{U}_{0}$ \\
\hline 9 & Loop $\mathrm{n}=1:$ Iter (Levenberg-Marquardt iteration $)$ \\
\hline 10 & compute $\mathbf{C}_{\mathbf{U}}$ and $\partial \mathbf{C}_{\mathbf{U}} / \partial \mathbf{U}$ \\
\hline 11 & compute the arrays $\mathbf{f}$ and $\mathbf{J}$ \\
\hline 12 & compute the parameters $\gamma_{1}$ and $\gamma_{2}$ from Eq. 7 \\
\hline 13 & compute the velocity step $\mathbf{U}_{l m}$ from Eq. 6 \\
\hline 14 & update the velocity $\mathbf{U}$ as given in Eq. 6 \\
\hline 15 & Perform subpixel interpolation by means of Gaussian fitting \\
\hline 16 & End \\
\hline
\end{tabular}


However, it is inefficient in solving a high-dimensional system of linear equations. To balance the computation time and density of the vector field, the step size is proposed to be between 2 and 6 pixels. A detailed time comparison is given in Sect. 4 for a real PIV experiment. The GOCCPIV method is 5.8 times faster than FFTCCPIV in terms of the time cost per vector. In GOCCPIV, a fixed number of optimization iterations (Iter $=10)$ is adopted because the convergence of the Levenberg-Marquardt method is very fast. Compared to the original PIV algorithm, another potential benefit of GOCCPIV is that the GOCCPIV algorithm can be implemented on a GPU device with high parallelization to further greatly reduce the computation time (Champagnat et al. 2011).

\subsection{Image pyramids for large displacements}

As stated in Sect. 2.1, due to the limitations of the optimization algorithm, GOCCPIV can find the correlation peaks correctly only if the displacement is less than 2-3 pixels (when the initial field is zero). However, in practice, larger displacements are common in PIV experiments. There are two ways to address this problem. One is to calculate the displacement from two successive images using the original PIV algorithm and then use GOCCPIV to refine the velocity field based on the small displacement after image deformation. This method is similar to that proposed by Seong et al. (2019) where the velocity is refined based on the global optical flow with a constant brightness gradient. The second method, which is applied in this work, is to construct multiresolution image pyramids from coarser to finer resolutions, as performed in optical flow analysis (Burt and Adelson 1983; Champagnat et al. 2011; Cassisa et al. 2011). At pyramid level $k$, the image is obtained by applying a low-pass filter and downsampling by a factor of 2 to the image at level $k-1$. Figure 4 shows such a multiresolution scheme with $K=3$, where $K$ is the total number of layers of the image pyramid. $K$ is determined from the maximum displacement as follows: $2^{K} \leq \max (\mathbf{U})$. The image resolution

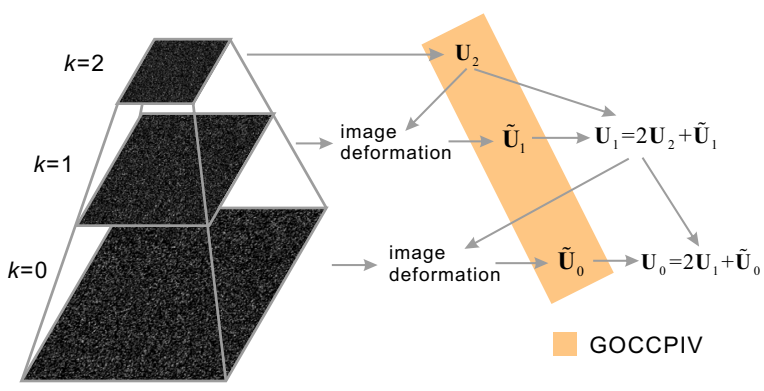

Fig. 4 Flowchart of iterative image deformation with GOCCPIV in a three-level image pyramid is reduced as the level $k$ increases. At pyramid level $k$, the velocity field is $\mathbf{U}_{k}=2 \mathbf{U}_{k+1}+\tilde{\mathbf{U}}_{k}$, where $\tilde{\mathbf{U}}_{k}$ is the incremental velocity field estimated from the deformed images at the current level. The detailed steps are as follows:

1. Create the image pyramids from level 0 (original images) to level $K-1$ (coarsest images).

2. Estimate the displacement $\mathbf{U}_{k}$ at level $k=K-1$ using GOCCPIV with zero initialization.

3. Deform images A and B at pyramid level $k$ in accordance with the velocity field $2 \mathbf{U}_{k+1}$. Both images A and $\mathrm{B}$ are deformed to the center time in accordance with the interpolated displacements obtained with the center difference pattern.

4. Estimate the incremental velocity field $\tilde{\mathbf{U}}_{k}$ from the deformed images using GOCCPIV with zero initialization.

5. Refine the velocity field at level $k$ as follows: $\mathbf{U}_{k}=2 \mathbf{U}_{k+1}+\tilde{\mathbf{U}}_{k}$.

6. Repeat steps 3 to 5 until $k=0$.

\section{Numerical evaluation}

\subsection{Synthetic images}

The performance of the proposed method is first evaluated using synthetic images generated in accordance with the framework of the EUROPIV Synthetic Image Generator (SIG) proposed by Lecordier and Westerweel (2004). The CCD resolution is set to $512 \times 512$ pixels and the ratio of the sensitive area of one pixel to the pixel pitch is fixed at 1. The intensity distribution is calculated by integrating the 2D Gaussian intensity distribution over the pixel area with a particle image diameter $d_{p}$ and a standard deviation of 0.2 pixels (Sciacchitano et al. 2013). Unless otherwise indicated, the number of particles per pixel ppp and particle diameter $d_{p}$ are set to 0.1 and 2 pixels, respectively, and $10 \%$ out-ofplane motion is applied to the particles. The peak intensity level of the particles is 255 , with a standard deviation of 50 counts. Noisy images are generated by adding Gaussian white noise with different standard variances to the original images. The noise level $\epsilon_{\text {noise }}$ is defined as the ratio between the standard variance of the noise and the maximum image intensity. The intensity values of the particle images are converted into 8-bit unsigned integers. Figure 5 displays two examples of synthetic particle images. The left is generated in accordance with $p p p=0.1$ and $d_{p}=1$ pixel without noise, and the right is generated with $p p p=0.1$ and $d_{p}=2$ pixels with Gaussian white noise of $\epsilon_{\text {noise }}=10 \%$.

The results of the proposed global optimization method will be compared with those of the WIDIM technique (Scarano 2002) and physics-based optical flow (Liu 2017; Liu et al. 


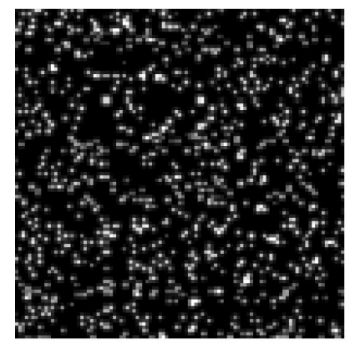

(a)

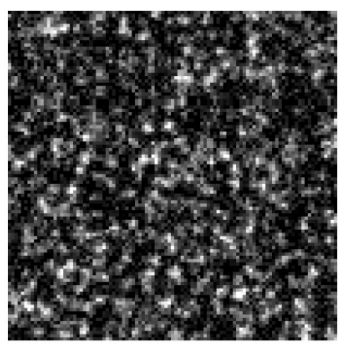

(b)
Fig. 5 Synthetic particle images with size of $100 \times 100$ pixels. a $p p p$ $=0.1$, particle diameter $d_{p}=1$ pixel without noise. $\mathbf{b} p p p=0.1, d_{p}=$ 2 pixels with Gaussian white noise at a level of $\epsilon_{\text {noise }}=10 \%$

2020). Because the WIDIM method is based on FFT-based cross-correlation, we refer to this method as FFTCCPIV. The physics-based optical flow is referred to as OpticalFlow. For FFTCCPIV, the number of iterations of image deformation is denoted by $N_{D}$. If $N_{D}$ is equal to 1 , this implies that only a single-pass cross-correlation is adopted in FFTCCPIV. If $N_{D}$ is larger than 1 , an iterative analysis at a fixed IW size is utilized to perform image deformation (Schrijer and Scarano 2008). A square IW is adopted for both FFTCCPIV and GOCCPIV to estimate the velocity fields. The open Matlab programs of OpticalFlow can be downloaded from https://github.com/Tians hu-Liu/OpenOpticalFlow. This approach is actually a hybrid optical-flow-cross-correlation method (Liu et al. 2020). The cross-correlation method is used to estimate a coarse-grained displacement field, and then, an optical flow estimation with the Horn-Schunck estimator and Liu-Shen estimator is applied to refine the field based on deformed images. Therefore, the parameters used in OpticalFlow contain the IW size for the PIV algorithm and Lagrange multipliers for optical flow estimators. The default parameters in the open source program in Matlab are adopted except that the filter size for the image is modified to 3. A statistical analysis is conducted by considering the root mean square error (RMSE) and average angular error [AAE, Barron et al. (1994)] between the calculated field $\mathbf{U}_{c}$ and the exact field $\mathbf{U}_{e}$ as follows:

$$
\begin{aligned}
\delta_{R M S E} & =\sqrt{\frac{1}{N} \sum_{x \in \Omega}\left(\mathbf{U}_{c}(x)-\mathbf{U}_{e}(x)\right)^{2}}, \\
\delta_{A A E} & =\frac{1}{N} \sum_{x \in \Omega}\left\{\arccos \left(\frac{\mathbf{U}_{c}(x)}{\left|\mathbf{U}_{c}(x)\right|} \cdot \frac{\mathbf{U}_{e}(x)}{\left|\mathbf{U}_{e}(x)\right|}\right)\right\},
\end{aligned}
$$

where $|\mathbf{U}|$ represents the velocity length of $\mathbf{U}$, the operator - represents the inner product, $N$ is the number of grid nodes of the velocity field (excluding the boundary) over the measurement region $\Omega$ and $x$ represents the vector locations.

\subsection{One-dimensional sinusoidal shear displacement}

One-dimensional sinusoidal shear displacement is considered to evaluate the performance of the proposed GOCCPIV algorithm as well as FFTCCPIV and OpticalFlow. The amplitude of the ground-truth velocity field $U_{0}$ varies from 0 to 8 pixels, and the wavelength $\lambda$ is set to 80 pixels. To draw a fair comparison of the effect of the IW size, particle concentration $p p p$, and particle image diameter $d_{p}$, the number of image pyramid levels $K$ is set to 1, meaning that only the original image pairs are used to evaluate the displacement in GOCCPIV. A standard single-pass correlation without image deformation is adopted in FFTCCPIV. For comparison of the effect of maximum particle displacement $U_{0}$, three image pyramid levels $(K=3)$ are adopted for GOCCPIV, and image deformation with $N_{D}=3$ is used within FFTCCPIV.

Figure 6 presents the displacement distributions obtained with FFTCCPIV (a, b), GOCCPIV (c, d) and OpticalFlow (e, f) with and without image noise for $\lambda=80$ pixels and $U_{0}=2$ pixels. The IW size and step size for both FFTCCPIV and GOCCPIV are 5 pixels and 2 pixels, respectively. Gaussian white noise with $\epsilon_{\text {noise }}=10 \%$ has been added to the images in the right panels of Fig. $6 \mathrm{~b}, \mathrm{~d}$ and $\mathrm{f}$, while no image noise is present in Fig. 6a, c and e. The number of image pyramid levels, $K$, is equal to 1 due to the small maximum displacement for GOCCPIV, and no image deformation is adopted in FFTCCPIV. Note that the spurious vectors detected by the normalized median test (Westerweel et al. 2004) are removed from the velocity fields, so some locations have no red vectors. In the case of images without noise, the displacement distribution of GOCCPIV is almost the same as that of FFTCCPIV, and both results present good agreement with the ground truth. The outlier rates for FFTCCPIV and GOCCPIV are approximately $12 \%$ and $4 \%$. For the image with noise, the result of GOCCPIV is much better than that of FFTCCPIV, as presented in Fig. 6b, d. Approximately 60 percent of the vectors of FFTCCPIV are outliers due to the small IW and image noise, while this value for GOCCPIV is as small as $6 \%$. The mean profile of GOCCPIV presents good agreement with the ground truth both with and without image noise. The RMSEs (red dashed line) of FFTCCPIV, GOCCPIV and OpticalFlow are approximately 0.7 pixels, 0.3 pixels and 0.16 pixels when considering image noise, respectively. The velocity field obtained by OpticalFlow is smoother than the others and performs best among all three methods. In this comparison, the performance of GOCCPIV is slightly worse than that of OpticalFlow while much better than that of FFTCCPIV.

The influences of the IW size on the outlier rate and RMSE are presented in Fig. 7a and b, respectively. The outlier rate is less than $3 \%$ even considering the image noise of $\epsilon_{\text {noise }}=10 \%$ when the IW size is larger than 10 

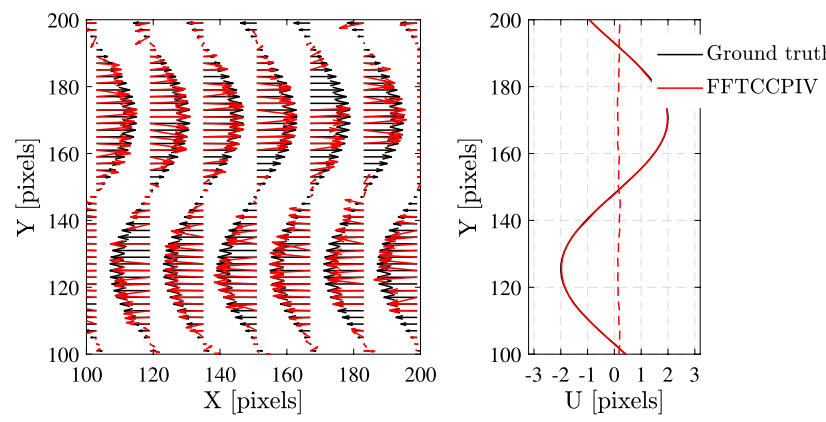

(a)
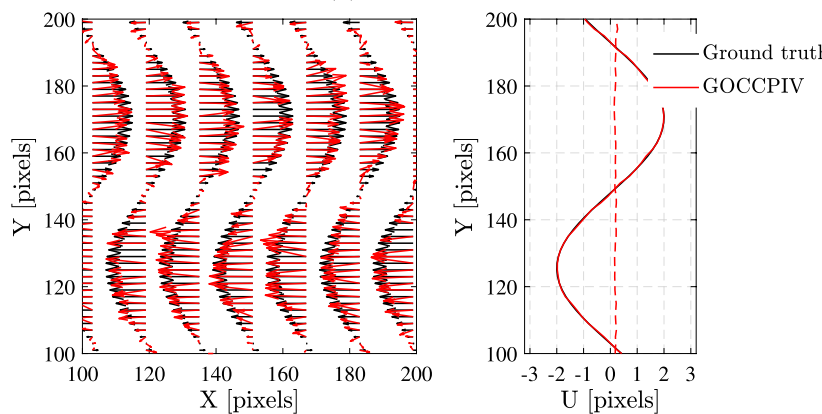

(c)

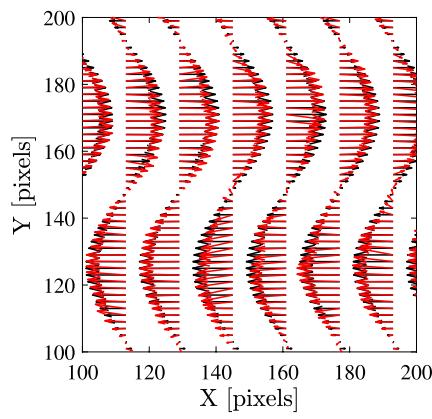

(e)
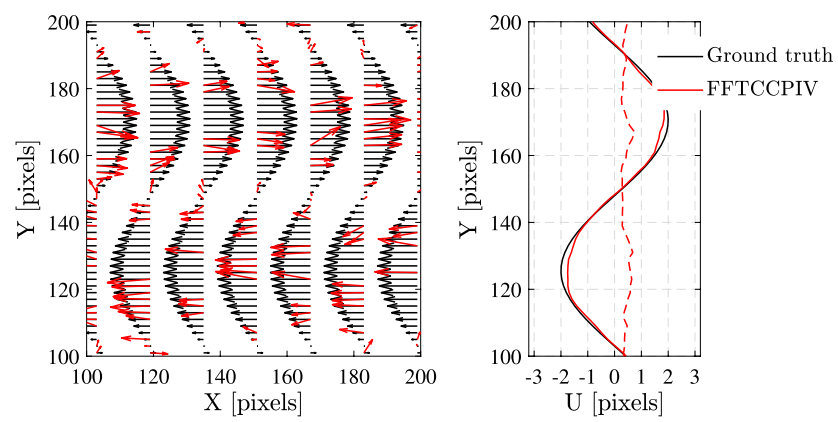

(b)
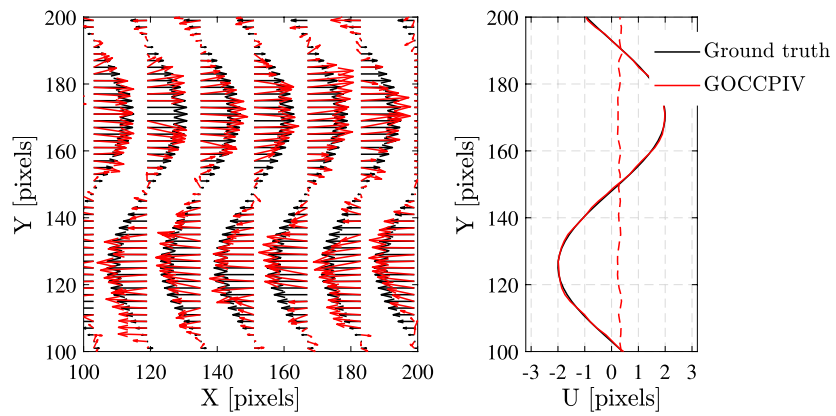

(d)
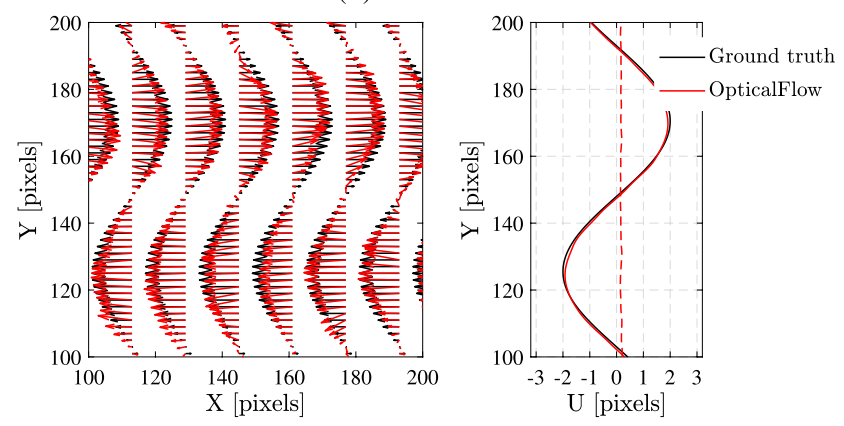

(f)
Fig. 6 Displacement distributions of FFTCCPIV (a, b), GOCCPIV $(\mathbf{c}, \mathbf{d})$ and OpticalFlow $(\mathbf{e}, \mathbf{f})$ for sinusoidal shear displacement with a wavelength of $\lambda=80$ pixels and $U_{0}=2$ pixels. The IW size for FFTCCPIV and GOCCPIV are 5 pixels, and the step size is 2 pixels. The left panels show the results without image noise, and the right panels show the results with image noise at a level of $\epsilon_{\text {noise }}=10 \%$. The left side of each panel shows the vector plot (vectors are displayed every $8 \times 8$ pixels), and the right side shows the mean sinusoidal curves. The solid black and red lines represent the ground-truth and calculated values, respectively. The dashed red lines represent the

pixels. According to the given $p p p$ of 0.1 , there are 10 particle image pairs within the IW of $10 \times 10$ pixels. This result confirms that the IW should contain sufficient particles (generally greater than 10) to ensure a reliable correlation peak (Adrian 1991; Scharnowski and Kähler 2020). The outlier rate dramatically increases when the IW size is smaller than 10 pixels for FFTCCPIV. Especially for the images containing noise, the outlier rate of FFTCCPIV reaches approximately $60 \%$ when the IW size decreases to 5 pixels. The outlier rate of GOCCPIV, by contrast, is less
RMSE estimated using Eq. 9. a Velocity field calculated via FFTCCPIV with an IW size of 5 pixels and no image noise. b Velocity field calculated via FFTCCPIV with an IW size of 5 pixels and image noise at a level of $\epsilon_{\text {noise }}=10 \%$. $\mathbf{c}$ Velocity field calculated via GOCCPIV with an IW size of 5 pixels and no image noise. $\mathbf{d}$ Velocity field calculated via GOCCPIV with an IW size of 5 pixels and image noise at a level of $\epsilon_{\text {noise }}=10 \%$. e Velocity field calculated via OpticalFlow without image noise. f Velocity field calculated via OpticalFlow with image noise at a level of $\epsilon_{\text {noise }}=10 \%$. The missing red vectors are detected outliers

than $6 \%$ in the whole range. The RMSEs as a function of IW size are shown in Fig. 7b. The errors of OpticalFlow are also indicated as the lines with square symbols in this figure. As the IW decreases in size from 30 pixels to 5 pixels, the RMSEs of both GOCCPIV and FFTCCPIV first decrease and then increase. When the IW is larger than 20 pixels, the performances of these two methods are comparable. When the IW is smaller than 20 pixels, the RMSE of GOCCPIV increases much more slowly than that of FFTCCPIV. This is because GOCCPIV uses a smoothness term 
Fig.7 Quantitative comparisons showing the effects of the IW size on the outlier rate (a) and error $\delta$ (b) for FFTCCPIV (circle), GOCCPIV (triangle) and OpticalFlow (square). Only a standard single-pass cross-correlation is adopted for $\operatorname{FFTCCPIV~}\left(N_{D}=1\right)$, and the image pyramid level of $K=1$ is adopted for GOCCPIV. The results without and with image noise are presented by solid and open symbols, respectively

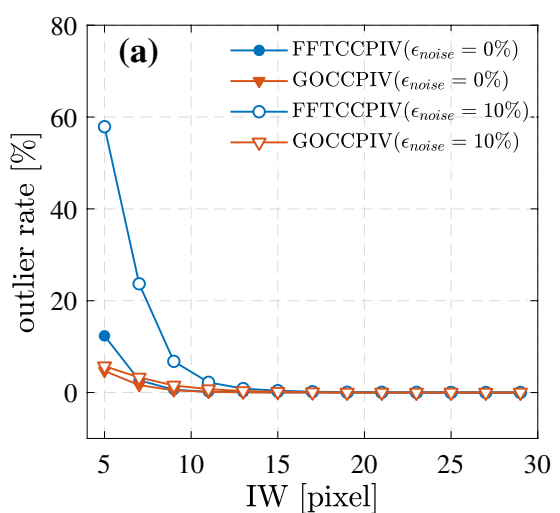

to optimize the velocity field. Considering the outlier rate given in Fig. 7a, most vectors are obtained by interpolation in FFTCCPIV for a small IW size. Moreover, the RMSE of OpticalFlow is smallest among these three methods.

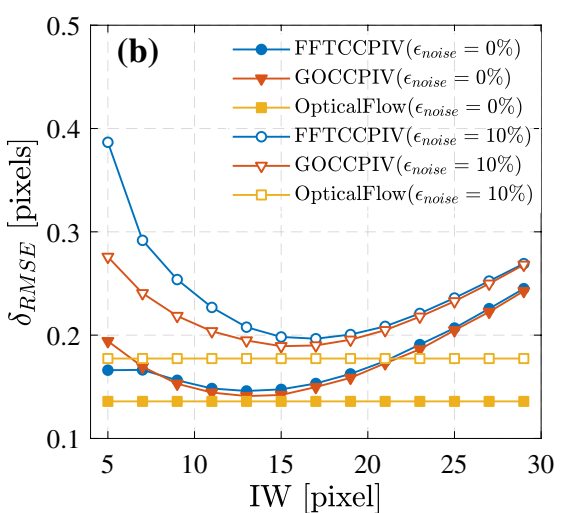

Detailed quantitative comparisons of the effects of particle concentration $p p p$, particle diameter $d_{p}$, maximum particle displacement $U_{0}$ and image noise level $\epsilon_{\text {noise }}$ are presented in Fig. 8. The top plane and bottom plane are the RMSE and AAE results, respectively. The curves with circles, triangles

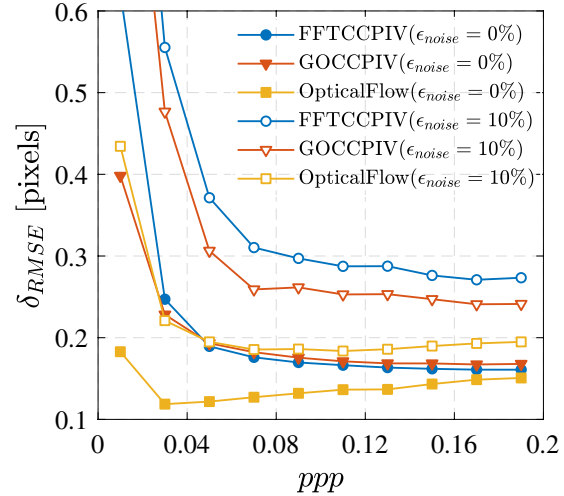

(a)

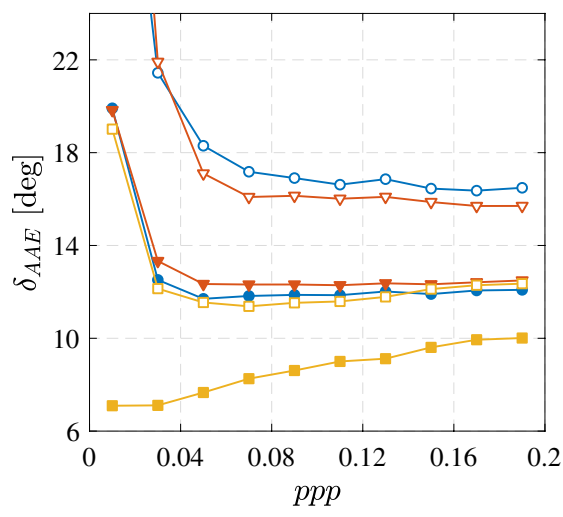

(d)

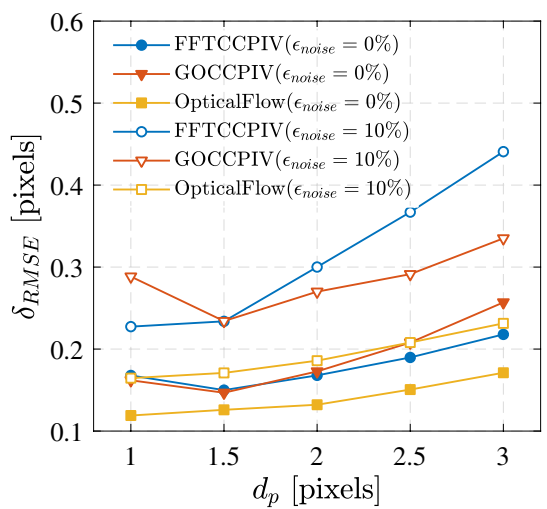

(b)

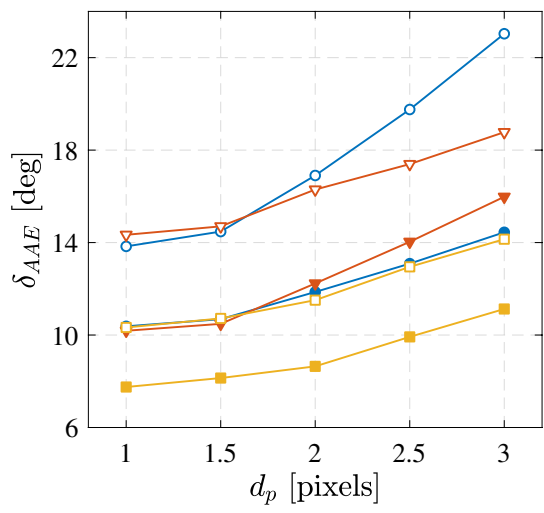

(e)

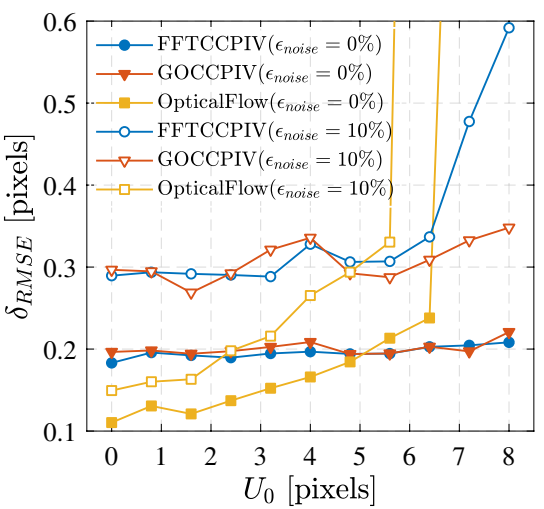

(c)

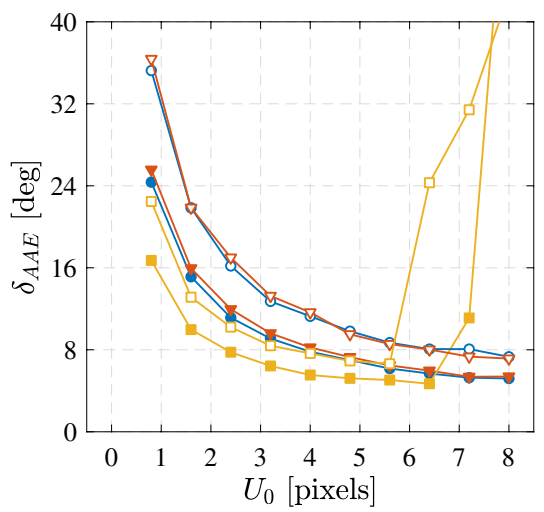

(f)
Fig. 8 RMSEs and AAEs as a function of particle concentration $p p p(\mathbf{a}, \mathbf{d})$, particle diameter $d_{p}(\mathbf{b}, \mathbf{e})$ and particle displacement $U_{0}$ $(\mathbf{c}, \mathbf{f})$ for FFTCCPIV (circle), GOCCPIV (triangle) and OpticalFlow (square). The results without and with image noise are presented by solid and open symbols, respectively. For figures $(\mathbf{a}, \mathbf{b}, \mathbf{d}, \mathbf{e})$, only a standard single-pass cross correlation is adopted for FFTCCPIV
$\left(N_{D}=1\right)$, and the image pyramid level of $K=1$ is adopted for GOCCPIV. For figure (c, f), image deformation with $N_{D}=3$ is used within FFTCCPIV, and three image pyramid levels $(K=3)$ are adopted for GOCCPIV. For all cases, the IW size and step size are 7 pixels and 2 pixels, respectively 
and squares represent the results of FFTCCPIV, GOCCPIV and OpticalFlow, respectively. In Fig. 8a, the RMSEs of FFTCCPIV and GOCCPIV present exponential decay as ppp increases from 0.01 to 0.2 . The error level of GOCCPIV is smaller than that of FFTCCPIV when considering image noise. It can be observed that the RMSE of OpticalFlow first decreases and then increases with increasing $p p p$. The effect of $d_{p}$ is investigated in Fig. 8b. An optimal particle diameter is approximately $d_{p}=1.5$ pixels for both FFTCCPIV and GOCCPIV. This is consistent with known results from the reports of Sciacchitano et al. (2013) and Wieneke (2015). The error of OpticalFlow monotonically increases as the particle diameter increases. We also consider the effect of the particle displacement $U_{0}$ in Fig. 8c. To assess the effect of a large displacement, three image pyramid levels $(K=3)$ are adopted for GOCCPIV. For FFTCCPIV, image deformation with $N_{D}=3$ is used to resolve the velocity fields. The IW sizes of GOCCPIV and FFTCCPIV are both 7 pixels, and the step size is 2 pixels. We compare the RMSEs for GOCCPIV and FFTCCPIV with and without image noise as the maximum displacement increases from 0 pixels to 8 pixels. The RMSE of GOCCPIV is almost the same as that of FFTCCPIV in the range of 0 pixels to 6 pixels. When $U_{0}$ is larger than 6 pixels, the performance of GOCCPIV is much better than that of FFTCCPIV when considering image noise. OpticalFlow outperforms FFTCCPIV and GOCCPIV at small displacement $\left(U_{0} \leq 5\right.$ pixels) but deteriorates with larger displacement (Liu et al. 2015; Seong et al. 2019). Figure $8 \mathrm{~d}-\mathrm{f}$ shows the AAE as a function of $p p p, d_{p}$ and $U_{0}$, respectively. The effects of $p p p$ on the AAE are almost the same as those on the RMSE, as are the effects of $d_{p}$. However, in Fig. 8f , the AAE decreases as $U_{0}$ increases. This is because the definition of the AAE in Eq. 9 is relative to $U_{0}$.
At the end of this part, we would like to give two remarks about the performance of GOCCPIV. First, GOCCPIV has the capability to resolve the velocity using a very small IW, which breaks the rule of "ten particles in the IW" for conventional FFTCCPIV. Second, GOCCPIV outperforms FFTCCPIV, while the performance of OpticalFlow is the best among all three methods under some conditions.

\subsection{Two-dimensional sinusoidal vortices}

An analysis of the spatial resolution is performed on test images of two-dimensional sinusoidal vortices. This test case was provided in the third PIV challenge, and the purpose of this case is to quantitatively determine the spatial resolution of the different methods. The images were downloaded from the challenge website (http://www.pivchallenge.org/) and have been described in detail in the literature by Stanislas et al. (2008). We use only the lower-left $1000 \times 1000$ pixels of the image region, which presents a set of random two-dimensional sinusoidal vortices. According to Stanislas et al. (2008), the sinusoids have different wavelengths varying from 8 to 200 pixels, and the amplitude varies around 2 pixels. Figure 9 illustrates the resolution test. From left to right, the V-component displacement fields evaluated by FFTCCPIV, GOCCPIV and OpticalFlow are displayed in Fig. $9 \mathrm{a}-\mathrm{c}$, respectively. Image deformation with $N_{D}=3$ is adopted for FFTCCPIV with an IW size of 32 pixels and $75 \%$ overlap. GOCCPIV with an IW size of 5 pixels and a step size of 2 pixels is used to evaluate the velocity field. The number of pyramid levels, $K$, is set to 2 . All large-scale vortices are well resolved by these three approaches. However, GOCCPIV recovers more small flow structures without introducing significant errors. From visual inspection of the

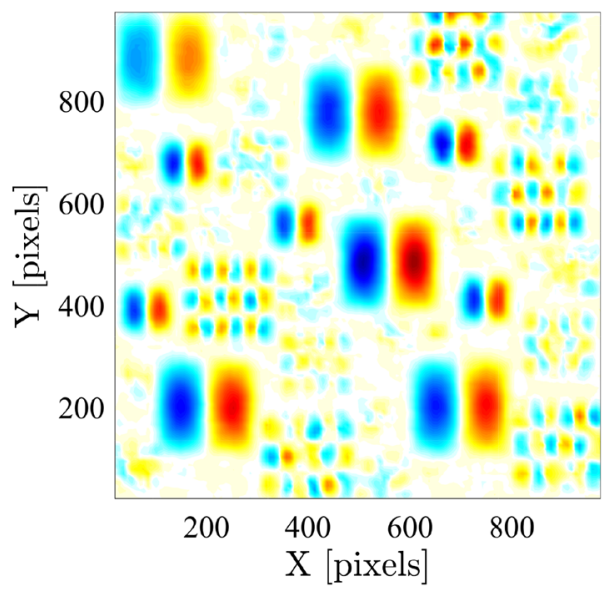

(a)

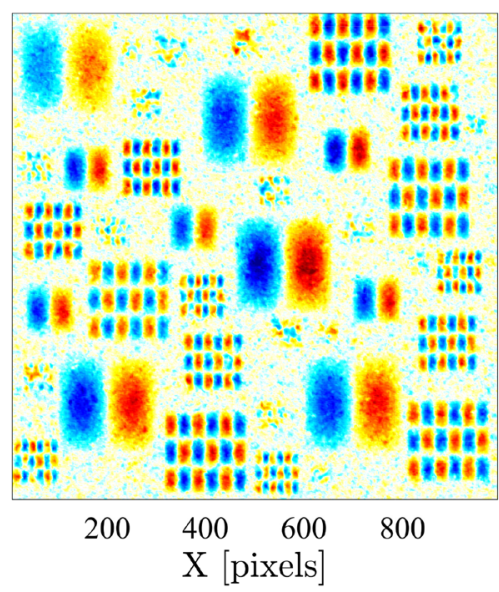

(b)

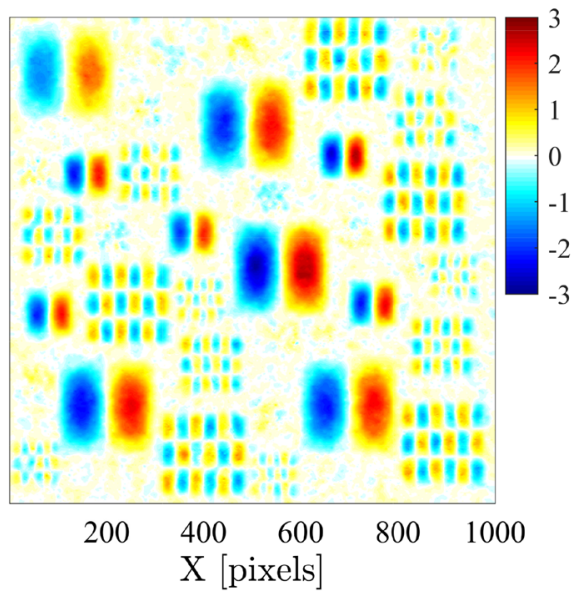

(c)

Fig. 9 V-component contour maps of two-dimensional sinusoidal vortices calculated via FFTCCPIV with $N_{D}=3$, IW size of 32 pixels and $75 \%$ overlap (a), GOCCPIV with $K=2$, IW size of 5 pixels and step size of 2 pixels (b), and OpticalFlow (c) 
contour of OpticalFlow, the small-scale vortices are visible, but the magnitude of the $\mathrm{V}$-component is much less than in the result of GOCCPIV. Both the results of FFTCCPIV and OpticalFlow are oversmoothed compared with GOCCPIV.

\subsection{Two-dimensional homogeneous turbulence}

A 2D homogeneous and isotropic turbulent flow is also used to evaluate the performance of the proposed method. The data sequence was downloaded online from http://fluid.irisa .fr/data-eng.htm, and a detailed description has been given by Carlier and Wieneke (2005). Fifty snapshots are used to generate synthetic images with a resolution of $512 \times 512$ pixels. The maximum displacement is set to 6 pixels. Gaussian white noise with a level of $5 \%$ is added to all synthetic images to assess the influence of noise on the proposed method.
Figure 10 displays instantaneous vortex maps (top) and error distributions (bottom) calculated using FFTCCPIV (a, d), GOCCPIV (b, e) and OpticalFlow (c, f). For FFTCCPIV, the number of iterations of image deformation is $N_{D}=3$, and the IW size is 32 pixels with $75 \%$ overlap. For GOCCPIV, the number of pyramid levels is set to 3 , and the IW size and step size are 9 pixels and 2 pixels. The error shown in Fig. 10 is defined as follows:

$e(i, j)=\sqrt{\left[u(i, j)-u_{r e f}(i, j)\right]^{2}+\left[v(i, j)-v_{r e f}(i, j)\right]^{2}}$,

where $u_{r e f}$ and $v_{r e f}$ are the exact reference u-component and $\mathrm{v}$-component, respectively, and the parameters $i$ and $j$ are the vector indices for different locations. In Fig. 10d, the largest errors always appear in regions with a high velocity gradient due to the modulation effect caused by the large IW (32 pixels). The mean error in Fig. 10d is 0.21 pixels. A weak modulation effect is still observed for OpticalFlow

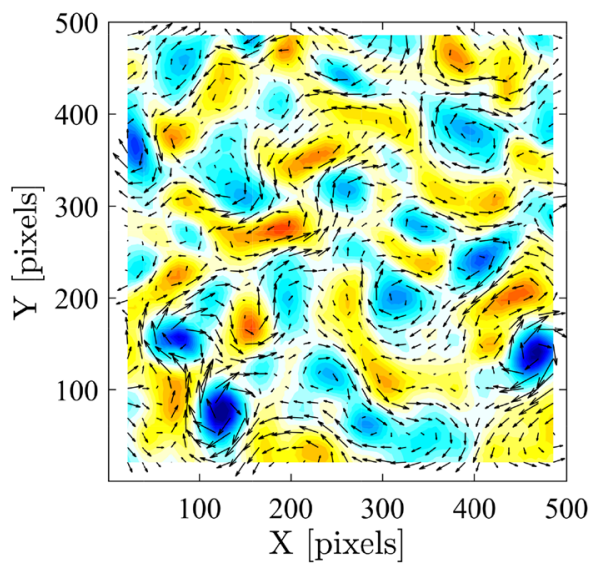

(a)

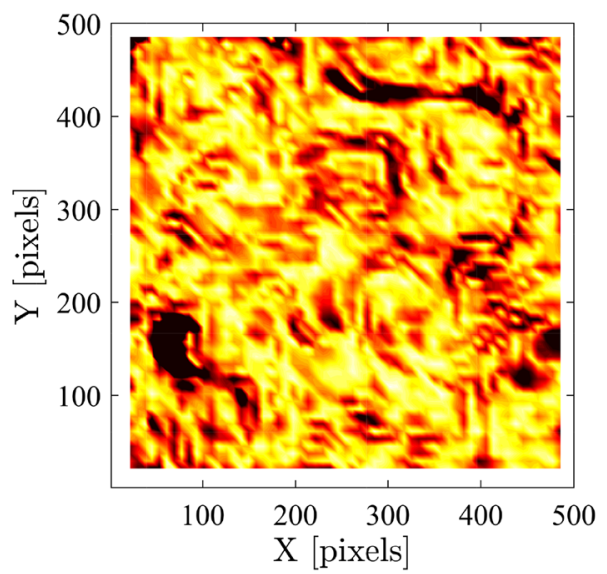

(d)

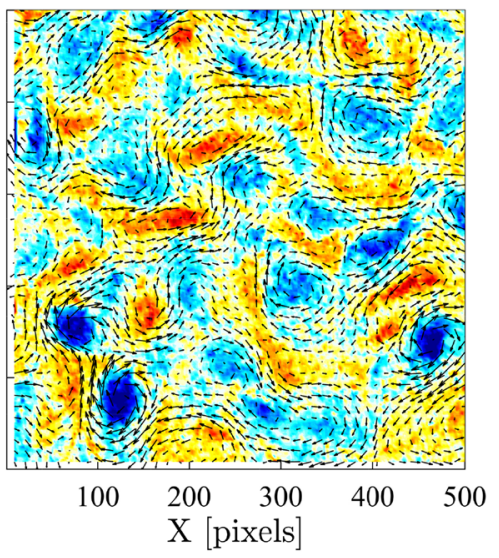

(b)

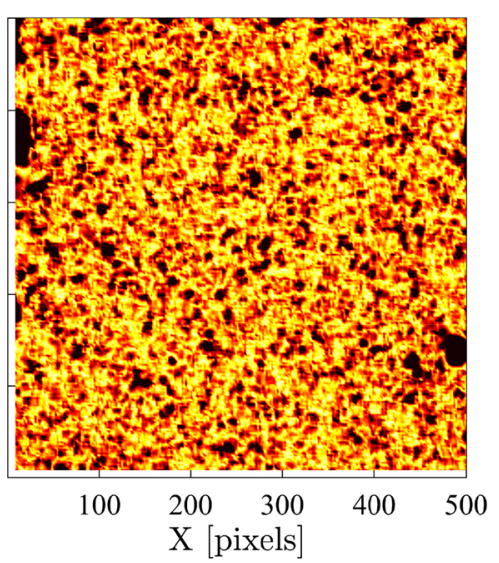

(e)

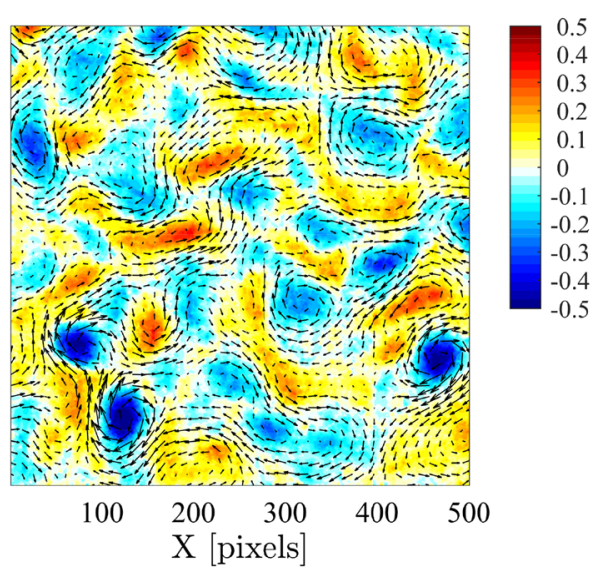

(c)

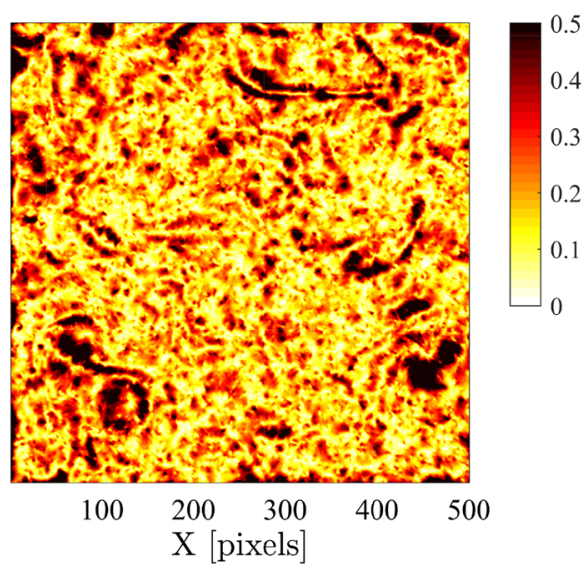

(f)
Fig. 10 Instantaneous raw vortex fields (top) and corresponding error distributions (bottom) for an image with 5\% noise. The left panels (a, d) show the results of FFTCCPIV with $N_{D}=3$, IW size of 32 pixels and $75 \%$ overlap, the center panels $(\mathbf{b}, \mathbf{e})$ show the results of GOC-
CPIV with $K=3$, IW size of 9 pixels and step size of 2 pixels, and the right panels $(\mathbf{c}, \mathbf{f})$ show the results of OpticalFlow. Filled contour maps are presented to display the vortex field and the error magnitude. All computed velocities are raw data without smoothing 
in Fig. 10f, and the mean error is 0.21 pixels, the same as that of FFTCCPIV. By contrast, in the GOCCPIV results shown in Fig. 10e, no modulation effect is evident, and the influence of random noise is acceptable. The mean error is approximately 0.24 pixels. Note that some large errors arise at the computation boundaries.

Figure 11 compares the results for the power spectral density (PSD) obtained from the velocity fields using FFTCCPIV, GOCCPIV and OpticalFlow. The images used in Fig. 11a have been generated with image noise, while images without any noise are also evaluated in Fig. 11b. $k$ represents the streamwise wavenumber. All spectra follow the same trend as the reference in the low-wavenumber domain. The energy spectrum of FFTCCPIV (IW=32 pixels) exhibits an obvious modulation effect in the high-wavenumber domain due to the large size of the IW (Foucaut et al. 2004; Atkinson et al. 2013). Although all spectra diverge from the reference spectrum, the spectra of GOCCPIV and OpticalFlow are much closer to the reference than that of FFTCCPIV. By carefully checking the spectrum of OpticalFlow, an underestimation can be observed in the range of $0.016 \leq k \leq 0.036$, which may be caused by a slightly strong smoothness constraint. The spectrum of GOCCPIV presents good agreement with the reference at $k \leq 0.03$, with a divergence toward higher energy at larger wavenumbers due to the random error, as shown in Fig.10e.

\section{Experiment with an impinging jet}

In this section, the proposed method is verified based on a real PIV experiment with an impinging jet. The impinging jet was formed as a synthetic jet vortex ring impinging on a solid wall (Xu et al. 2017). As shown in Fig. 12, this experiment was conducted in an acrylic water tank with dimensions of $600 \mathrm{~mm} \times 600 \mathrm{~mm} \times 600 \mathrm{~mm}$. The height of the water was $550 \mathrm{~mm}$ to ensure no boundary disturbance. An L-shaped hollow circular cylinder with an exit orifice diameter $D$ of $10 \mathrm{~mm}$ was placed under the water, and the exit orifice was approximately $300 \mathrm{~mm}$ from the sidewall and approximately $316 \mathrm{~mm}$ from the bottom of the tank. The L-shaped hollow cylinder was connected to a piston-cylinder
Fig. 11 Energy spectra of a two-dimensional turbulent flow obtained from images with (a) and without (b) noise. The computational configurations are the same as in Fig. 10

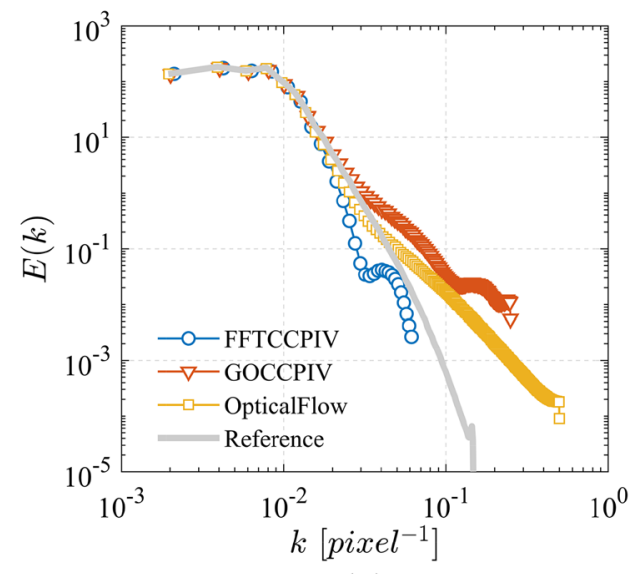

(a)

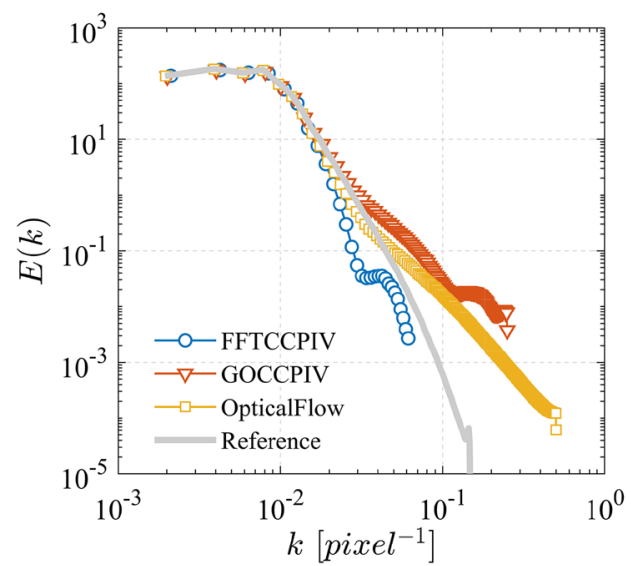

(b)
Fig. 12 Schematic diagram of the experimental setup

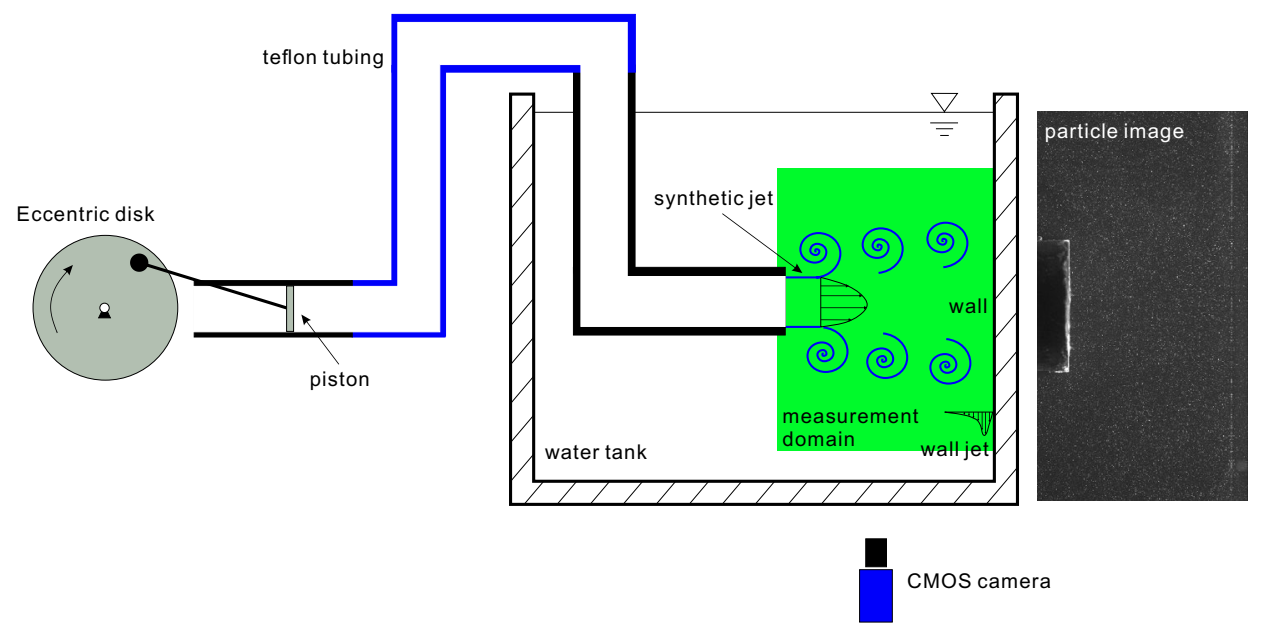


arrangement by Teflon tubing, and the piston was connected to an eccentric disk driven by a high-precision servo motor. A detailed description of the configuration used in this experiment can be found in the paper by Xu et al. (2017). After activation of the motor, a periodic synthetic jet perpendicularly impinges on the sidewall. In the present work, we consider only the case of $R e_{s j}=332$, where the Reynolds number $R e_{s j}$ is defined as $R e_{s j}=V_{s j} D / \nu$. The parameter $V_{s j}$ is the characteristic velocity of the synthetic jet, estimated as the time-averaged orifice blowing velocity over an entire cycle, and $v$ is the kinematic viscosity of the water, which was estimated at a temperature of $20^{\circ} \mathrm{C}$. According to the parameters given by $\mathrm{Xu}$ et al. (2017), $V_{s j}$ and the maximum orifice fluid velocity are $33.3 \mathrm{~mm} / \mathrm{s}$ and $104.6 \mathrm{~mm} / \mathrm{s}$, respectively.

A time-resolved PIV system was used to acquire the particle images (Xu et al. 2017). Hollow glass beads with a diameter of $10 \mu \mathrm{m}$ were seeded into the water tank as tracer particles. These particles were illuminated by a continuous laser sheet with a thickness of $1 \mathrm{~mm}$ at a power of $5 \mathrm{~W}$. The laser sheet was emitted across the centerline of the synthetic jet. A high-speed CMOS camera (FastcamSA2/86K-M3) with a sampling frequency of $240 \mathrm{~Hz}$ was used to capture the particle images, and the measurement region is represented by the green domain in Fig. 12. A raw particle image is also shown in this figure; its size is $1536 \times 836$ pixels, with approximately $0.06 \mathrm{~mm}$ per pixel, corresponding to a field of view of approximately $90 \mathrm{~mm} \times 50 \mathrm{~mm}$. The maximum particle displacement between adjacent snapshots is approximately 10 pixels, and the particles in the high-speed region exhibit a slight tailing effect due to the relatively long exposure time. Although many periods of vortex ring evolution were recorded, only one period of the jet is used here to analyze the performance of GOCCPIV. Before calculating the velocity field, the historical minimum value over the image sets is taken as an estimate of the background and subtracted from the raw image to reduce the image noise. The particle concentration $p p p$ is found to be approximately 0.0527 , corresponding to approximately fifty particles on average in an IW of 32 pixels $\times 32$ pixels. Note that, as indicated in Fig. 12, the synthetic jet region and wall jet region are challenging to analyze using the classical PIV algorithm due to the high-velocity gradient in these regions.

To validate GOCCPIV, the results of FFTCCPIV and OpticalFlow are also presented in this section. The IW of FFTCCPIV is set to the common dimensions of 32 pixels $x$ 32 pixels, and the number of iterations of image deformation is 3 . The step size is set to 8 pixels, corresponding to an overlap factor of $75 \%$. For GOCCPIV, because the maximum displacement is greater than 8 pixels, the number of pyramid layers, $K$, is set to 4 to generate the image pyramids. The IW size and step size of GOCCPIV are 9 pixels and 2 pixels, respectively. For OpticalFlow, the cross-correlation method with three passes $(64 \times 64,32 \times 32$ to $16 \times 16$ pixels with $50 \%$ overlap) is used to initialize the large displacement field, and then, the Horn-Schunck estimator with a Lagrange multiplier of 20 and Liu-Shen estimator with a Lagrange multiplier of 2000 are used to refine the flow field. The parameters used for each approach are listed in Table 2. No postprocessing is applied to the output velocity fields for either GOCCPIV or FFTCCPIV. The left side of one instantaneous velocity magnitude field computed via FFTCCPIV and GOCCPIV is presented in Fig. 13, and the contour map of GOCCPIV is plotted in mirror symmetry. The peak velocity of GOCCPIV is higher than that of FFTCCPIV, and the flow structure is well resolved via GOCCPIV with an increased spatial resolution.

Figure 14 shows the profiles of the v-component velocity at the center of the measurement domain as a function of

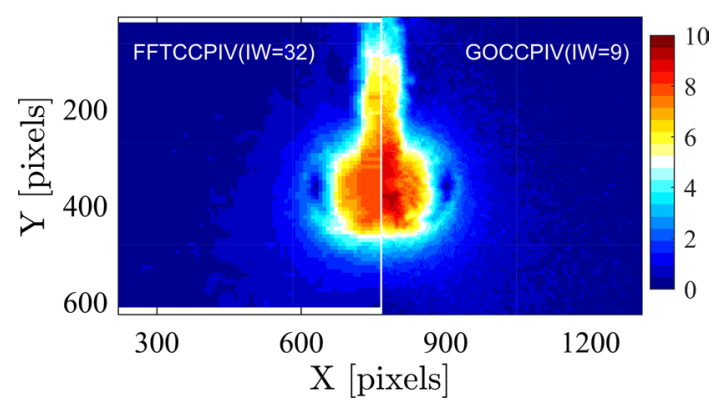

Fig. 13 Instantaneous velocity magnitude fields computed via FFTCCPIV (left) and GOCCPIV (right)

Table 2 PIV parameters and execution times for the impinging jet experiment

\begin{tabular}{lllllll}
\hline & IW size (pixels) & Step size (pixels) & $\begin{array}{l}\text { Number of iterations/ } \\
\text { levels }\end{array}$ & Number of vectors & CPU time (s) & Time per vector (s) \\
\hline FFTCCPIV & 32 & 8 & 3 & $75 \times 188$ & 14 & $9.9 \times 10^{-4}$ \\
GOCCPIV & 9 & 2 & 4 & $310 \times 760$ & 39 & $1.7 \times 10^{-4}$ \\
\hline & PIV parameters (pixels) & Step size (pixels) & Lagrange multipliers & Number of vectors & CPU time (s) & Time per vector (s) \\
\hline OpticalFlow & $(64,32,16)$ with 50\% overlap & 1 & $(20,2000)$ & $636 \times 1536$ & 28 & $0.29 \times 10^{-4}$ \\
\hline
\end{tabular}




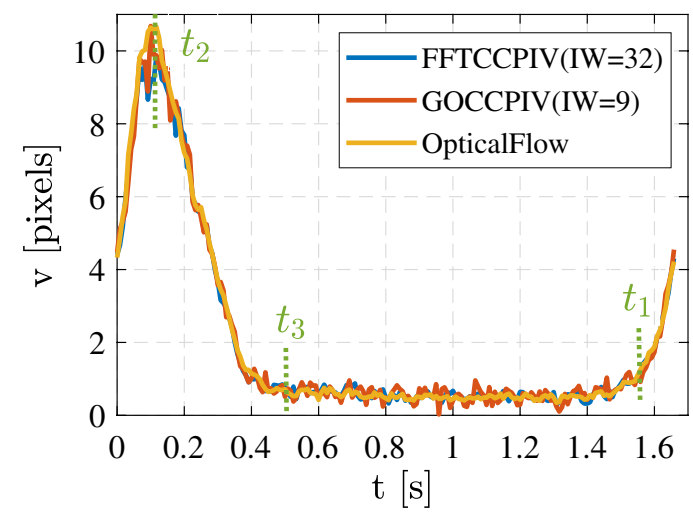

Fig. 14 Time evolution profiles of the v-component velocity at the center of the measurement domain. The blue, red and orange curves represent the results of FFTCCPIV, GOCCPIV and OpticalFlow, respectively. Three different flow states at different times are indicated by times $t_{1}, t_{2}$ and $t_{3}$

time obtained via FFTCCPIV, GOCCPIV and OpticalFlow. The v-component is perpendicular to and points toward the sidewall. One circle of the synthetic jet is shown in this figure. The peak velocity, which corresponds to the time when the jet passes through the center location, is higher in the profiles obtained with GOCCPIV and OpticalFlow than in that obtained with FFTCCPIV. The peak velocity of GOCCPIV is the same as that of OpticalFlow, except that the random fluctuations of the GOCCPIV profile are slightly larger than those of OpticalFlow. In this figure, three different flow states at different times are indicated by times $t_{1}$, $t_{2}$ and $t_{3}$. At time instant $t_{1}$, the vortex ring generated by the synthetic jet is close to the orifice exit and is moving toward the wall. At time $t_{2}$, the vortex ring is exactly in the center of the measurement domain. At time $t_{3}$, the impinging vortex ring is spread over the wall, leading to the generation of a secondary vortex ring and a tertiary vortex ring (Xu et al. 2017). The performance of the proposed method will be compared with FFTCCPIV and OpticalFlow at these three different instants of time.

The vorticity maps and vector distributions at times $t_{1}, t_{2}$ and $t_{3}$ obtained using the different methods are displayed in Fig. 15 from top to bottom. The contours show very good agreement in general, except for the higher random noise caused by the small IW in GOCCPIV. The smoothness degree of OpticalFlow is almost the same as that of FFTCCPIV with an IW size of 32 pixels. The specific region at each time instant is indicated by a gray dashed line, and quantitative comparisons at these locations are presented in Fig. 16. The horizontal lines at times $t_{1}$ and $t_{2}$ are located in the regions with a high velocity gradient and the center of the vortex ring, respectively. The vertical line at time $t_{3}$ is located in the wall jet region. From Fig. 16, the velocity is underestimated by FFTCCPIV due to the large IW. The results of GOCCPIV present good agreement with those of OpticalFlow in Fig. 16a, b. At the location of the wall jet as shown in Fig. 16c, the maximum u-component displacement of wall jet for OpticalFlow is larger than that for GOCCPIV, but, the profile of GOCCPIV is closer to the no-slip condition on the wall surface. Both the profiles of OpticalFlow and GOCCPIV present a higher velocity gradient than that of FFTCCPIV. By contrast, the profile of GOCCPIV indicates an improvement in resolving the vortex structures near the wall. Furthermore, the computation times with the different approaches are listed in Table 2. Both programs were coded and tested on a laptop with one CPU core in MATLAB, and the code performance was not optimized. The efficiency of OpticalFlow is the highest among all three methods, approximately 34 times that of FFTCCPIV. Note that the overlap factor in FFTCCPIV is $75 \%$, which is much larger than the value of $50 \%$ in OpticalFlow. The efficiency of GOCCPIV is approximately 5.8 times that of FFTCCPIV according to the time costs per vector listed in Table 2 .

\section{Conclusions and future work}

In this paper, we propose a novel PIV algorithm, GOCCPIV, for the assessment of velocity fields with high spatial resolution from particle image pairs. This method uses an optimization algorithm to globally minimize an objective function consisting of a cross-correlation term and a penalty term for smoothness. An empirical smoothing parameter is also presented to suppress the noise caused by a small IW. With this penalty term, a very small IW can be used in GOCCPIV to calculate a dense velocity field with a high spatial resolution. The performance of the proposed method is compared with those of an FFT-based cross-correlation technique and a physics-based optical flow approach on synthetic image pairs representing one-dimensional sinusoidal shear displacement, two-dimensional sinusoidal vortices and homogeneous turbulence. An error analysis considering the IW size, particle concentration, particle image diameter, large displacements and image noise indicates that GOCCPIV outperforms FFTCCPIV under identical conditions. OpticalFlow performs best among these three methods for small displacement. In particular, in the spatial resolution test based on two-dimensional sinusoidal vortices, the IW size in GOCCPIV can be reduced to 5 pixels to resolve more small-scale vortices, Both FFTCCPIV and OpticalFlow fail to resolve these small vortices. The test based on two-dimensional homogeneous turbulence shows that the modulation error of GOCCPIV is much smaller than that of conventional FFTCCPIV. An application of GOCCPIV to a real PIV experiment with an impinging jet flow shows that GOCCPIV can successfully resolve the velocity in the high-velocity-gradient regions of the synthetic jet and wall 


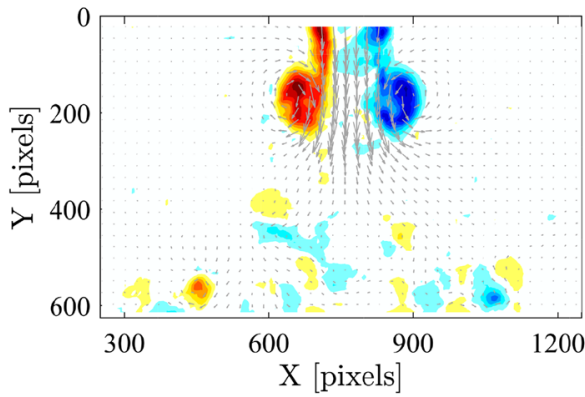

(a)

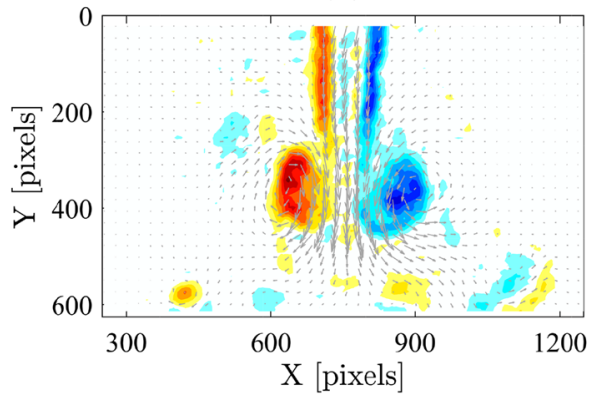

(d)

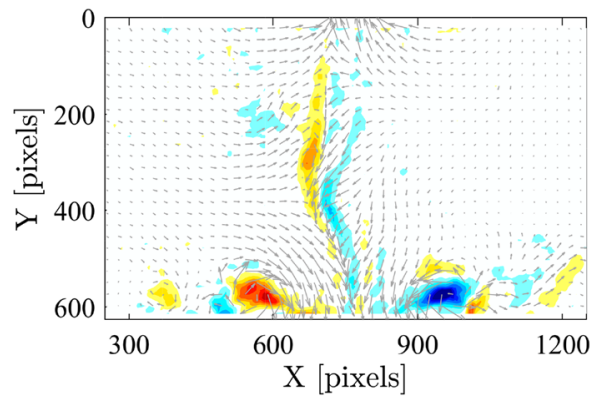

(g)

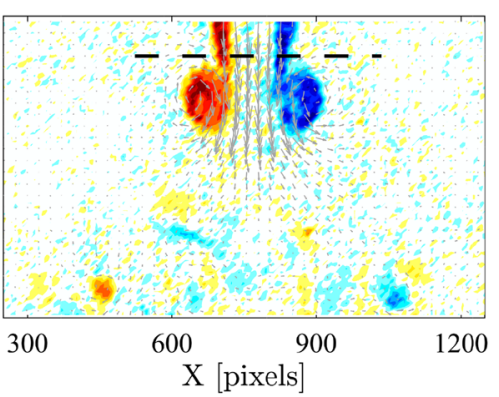

(b)

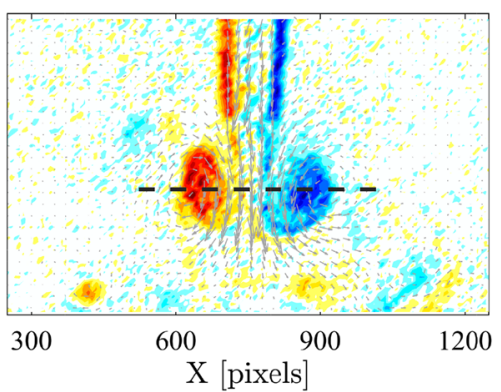

(e)

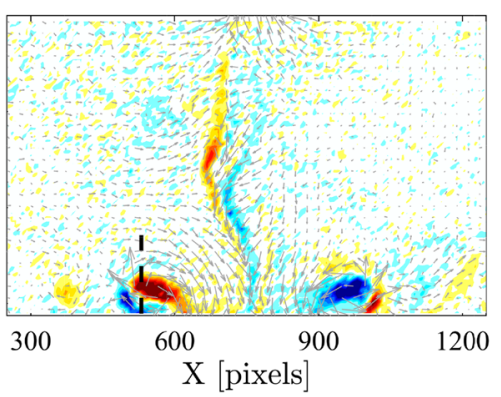

(h)

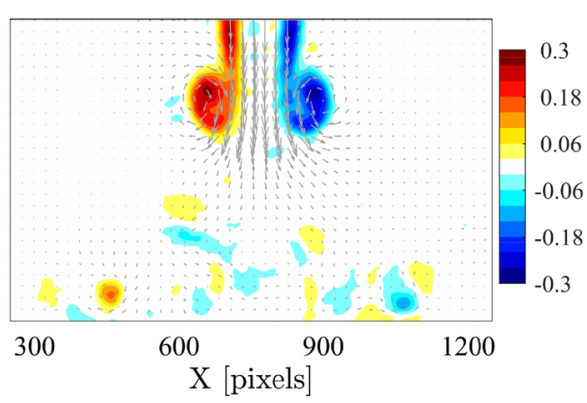

(c)

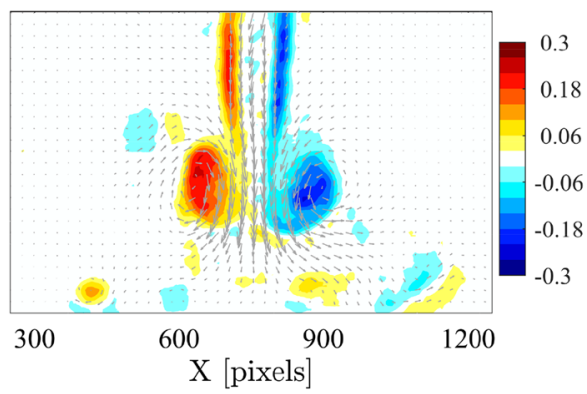

(f)

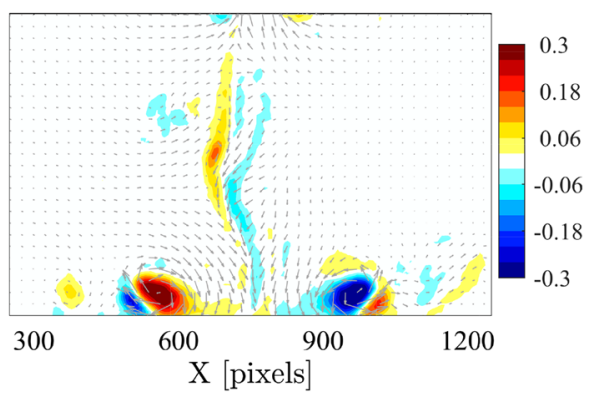

(i)
Fig. 15 Instantaneous vorticity maps and vector distributions obtained via FFTCCPIV with an IW size of 32 pixels (a, d, g), GOCCPIV with an IW size of 9 pixels $(\mathbf{b}, \mathbf{e}, \mathbf{h})$ and OpticalFlow $(\mathbf{c}, \mathbf{f}, \mathbf{i})$ at time instants $t_{1}(\mathbf{a}, \mathbf{b}, \mathbf{c}), t_{2}(\mathbf{d}, \mathbf{e}, \mathbf{f})$ and $t_{3}(\mathbf{g}, \mathbf{h}, \mathbf{i})$. For clarity, one in every three vectors, one in every twelve vectors and one in every 24 vectors are shown for FFTCCPIV, GOCCPIV and OpticalFlow, respectively. The gray dashed lines in $(\mathbf{b}, \mathbf{e}, \mathbf{h})$ represent the locations of the curves shown in Fig. 16

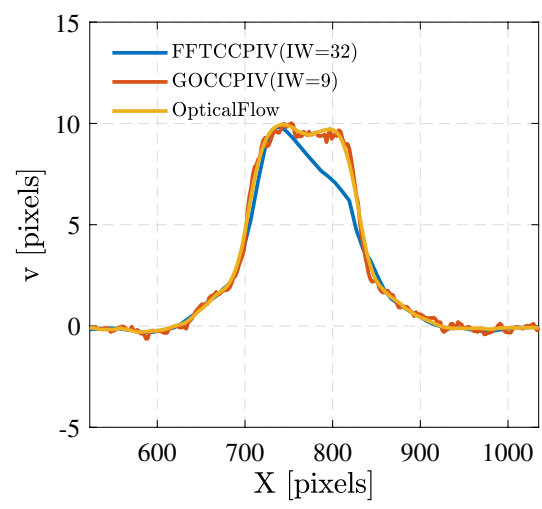

(a)

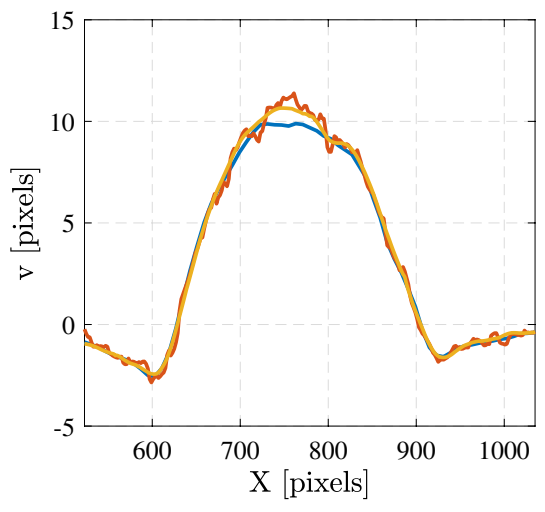

(b)

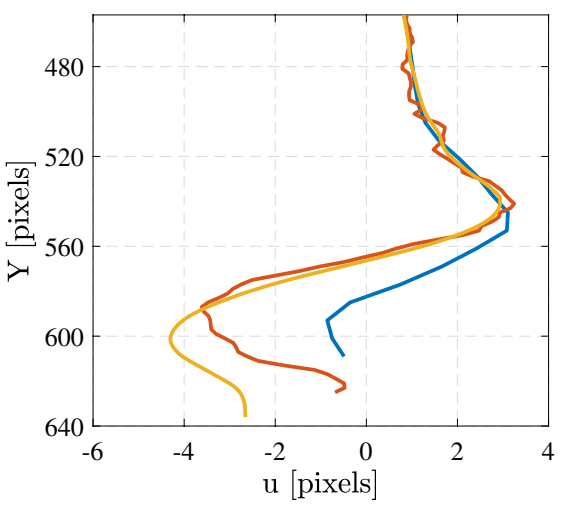

(c)

Fig. 16 Comparisons of the v-component displacement $(\mathbf{a}, \mathbf{b})$ and $\mathbf{u}$-component displacement $(\mathbf{c})$ profiles at the locations indicated by the gray dashed lines in Fig. 15. From left to right, the times are $t_{1}, t_{2}$ and $t_{3}$ 
jet compared with FFTCCPIV and OpticalFlow, and is more efficient than FFTCCPIV. Thus, the proposed method is useful for obtaining high-resolution velocity fields for data analysis and flow structure extraction.

We note that GOCCPIV can still be further improved in our future work. First, the random error of GOCCPIV must be further reduced. Most of this random error originates from the subpixel fitting procedure in the case of a small IW. Second, a physically based smoothness constraint on the penalty term will be required to accurately estimate flow motion. Third, the computational efficiency of GOCCPIV must be further improved. This can be achieved by developing parallelized GPU code. In brief, GOCCPIV is a novel way to obtain high-resolution, dense velocity fields.

Acknowledgements This work was supported by the NSFC Basic Science Center Program for 'Multiscale Problems in Nonlinear Mechanics' (Grant No. 11988102) and the National Natural Science Foundation of China (Grant Nos. 11702302, 11922214 and 91752118). The authors would also like to acknowledge the support from the Strategic Priority Research Program (Grant No. XDB22040104) and the Key Research Program of Frontier Sciences of the Chinese Academy of Sciences (Grant No. QYZDJ-SSW-SYS002). The authors gratefully acknowledge Yang Xu for providing the original experimental data.

\section{References}

Adamczyk AA, Rimai L (1988) 2-Dimensional particle tracking velocimetry (PTV): technique and image processing algorithms. Exp Fluids 6(6):373-380. https://doi.org/10.1007/BF00196482

Adrian RJ (1991) Particle-imaging techniques for experimental fluid mechanics. Ann Rev Fluid Mech 23(1):261-304. https://doi. org/10.1146/annurev.fl.23.010191.001401

Adrian RJ (2005) Twenty years of particle image velocimetry. Exp Fluids 39(2):159-169. https://doi.org/10.1007/s00348-005-0991-7

Atkinson C, Buchmann N, Amili O, Soria J (2013) On the appropriate filtering of PIV measurements of turbulent shear flows. Exp Fluids 55(1):1-15. https://doi.org/10.1007/s00348-013-1654-8

Barron JL, Fleet DJ, Beauchemin SS (1994) Performance of opticalflow techniques. Int J Comput Vis 12(1):43-77. https://doi. org/10.1007/bf01420984

Becker F, Wieneke B, Petra S, Schroder A, Schnorr C (2012) Variational adaptive correlation method for flow estimation. IEEE Trans Image Process 21(6):3053-3065. https://doi.org/10.1109/ tip.2011.2181524

Bruhn A, Weickert J, Schnorr C (2005) Lucas/kanade meets Horn/ Schunck: Combining local and global optic flow methods. Int $\mathrm{J}$ Comput Vis 61(3):211-231. https://doi.org/10.1023/B:VISI.00000 45324.43199 .43

Burt P, Adelson E (1983) The Laplacian Pyramid as a Compact Image Code. IEEE Trans Commun 31(4):532-540. https://doi. org/10.1109/TCOM.1983.1095851

Cai SZ, Zhou SC, Xu C, Gao Q (2019) Dense motion estimation of particle images via a convolutional neural network. Exp Fluids 60(4):73. https://doi.org/10.1007/s00348-019-2717-2

Carlier J, Wieneke B (2005) Report 1 on production and diffusion of fluid mechanics images and data. Fluid project deliverable 1.2. European Project 'Fluid image analisys and description' (FLUID)http://www fluid irisa fr 47
Cassisa C, Simoens S, Prinet V, Shao L (2011) Subgrid scale formulation of optical flow for the study of turbulent flow. Exp Fluids 51(6):1739-1754. https://doi.org/10.1007/s00348-011-1180-5

Champagnat F, Plyer A, Le Besnerais G, Leclaire B, Davoust S, Le Sant Y (2011) Fast and accurate PIV computation using highly parallel iterative correlation maximization. Exp Fluids 50(4):1169-1182. https://doi.org/10.1007/s00348-011-1054-x

Corpetti T, Memin E, Perez P (2002) Dense estimation of fluid flows. IEEE Trans Pattern Anal Mach Intell 24(3):365-380. https:// doi.org/10.1109/34.990137

Corpetti T, Heitz D, Arroyo G, Memin E, Santa-Cruz A (2006) Fluid experimental flow estimation based on an optical-flow scheme. Exp Fluids 40(1):80-97. https://doi.org/10.1007/s0034 8-005-0048-y

Drulea M, Nedevschi S (2013) Motion estimation using the correlation transform. IEEE Trans Image Process 22(8):3260-3270. https://doi.org/10.1109/tip.2013.2263149

Foucaut JM, Carlier J, Stanislas M (2004) PIV optimization for the study of turbulent flow using spectral analysis. Meas Sci Technol 15(6):1046-1058. https://doi.org/10.1088/0957-0233/15/6/003

Garcia D (2010) Robust smoothing of gridded data in one and higher dimensions with missing values. Comput Stat Data Anal 54(4):1167-1178. https://doi.org/10.1016/j.csda.2009.09.020

Garcia D (2011) A fast all-in-one method for automated post-processing of PIV data. Exp Fluids 50:1247-1259

Heitz D, Heas P, Memin E, Carlier J (2008) Dynamic consistent correlation-variational approach for robust optical flow estimation. Exp Fluids 45(4):595-608. https://doi.org/10.1007/s0034 8-008-0567-4

Horn BKP, Schunck BG (1981) Determining optical-flow. Artif Intell 17(1-3):185-203. https://doi.org/10.1016/0004-3702(81)90024 $-2$

Huang HT, Fiedler HE (1993) Deformed particle image pattern matching in particle image velocimetry. Appl Sci Res 51(1):179-183. https://doi.org/10.1007/bf01082534

Lecordier B, Westerweel J (2004) The EUROPIV synthetic image generator (SIG), Springer Berlin Heidelberg, book section 11, pp 145-161. https://doi.org/10.1007/978-3-642-18795-7_11

Lee Y, Yang H, Yin Z (2017) PIV-DCNN: cascaded deep convolutional neural networks for particle image velocimetry. Exp Fluids 58(12):171. https://doi.org/10.1007/s00348-017-2456-1

Levenberg K (1944) A method for the solution of certain problems in least-squares. Q Appl Math 2:164-168

Liu TS (2017) OpenOpticalFlow: an open source program for extraction of velocity fields from flow visualization images. J Open Res Softw. https://doi.org/10.5334/jors. 168

Liu TS, Shen LX (2008) Fluid flow and optical flow. J Fluid Mech 614:253-291. https://doi.org/10.1017/S0022112008003273

Liu TS, Merat A, Makhmalbaf MHM, Fajardo C, Merati P (2015) Comparison between optical flow and cross-correlation methods for extraction of velocity fields from particle images. Exp Fluids 56(8):23. https://doi.org/10.1007/s00348-015-2036-1

Liu TS, Salazar DM, Fagehi H, Ghazwani H, Montefort J, Merati P (2020) Hybrid optical-flow-cross-correlation method for particle image velocimetry. J Fluids Eng Trans ASME. https://doi. org/10.1115/1.4045572

Lucas BD, Kanade T (1981) An iterative image registration technique with an application to stereo vision. In: Proceedings Seventh International Joint Conference on Artificial Intelligence, pp 674-679

Maas HG, Gruen A, Papantoniou D (1993) Particle tracking velocimetry in three-dimensional flows. Exp Fluids 15:133-146

Marquardt D (1963) An algorithm for least-squares estimation of nonlinear parameters. SIAM J Appl Math 11:431-441

Nobach H (2011) Influence of individual variations of particle image intensities on high-resolution PIV. Exp Fluids 50(4):919-927. https://doi.org/10.1007/s00348-010-0948-3 
Nogueira J, Lecuona A, Rodriguez PA (1999) Local field correction PIV: on the increase of accuracy of digital PIV systems. Exp Fluids 27(2):107-116. https://doi.org/10.1007/s003480050335

Raffel M, Willert CE, Scarano F, Kahler CJ, Wereley ST, Kompenhans J (2018) Particle image velocimetry: a practical guide. Springer, New York

Scarano F (2002) Iterative image deformation methods in PIV. Meas Sci Technol 13(1):R1-R19. https://doi. org/10.1088/0957-0233/13/1/201

Scarano F (2004) A super-resolution particle image velocimetry interrogation approach by means of velocity second derivatives correlation. Meas Sci Technol 15(2):475-486. https://doi. org/10.1088/0957-0233/15/2/023

Scharnowski S, Kähler CJ (2020) Particle image velocimetry-classical operating rules from today's perspective. Opt Lasers Eng. https://doi.org/10.1016/j.optlaseng.2020.106185

Schneiders JFG, Scarano F (2016) Dense velocity reconstruction from tomographic PTV with material derivatives. Exp Fluids 57(9):122. https://doi.org/10.1007/s00348-016-2225-6

Schrijer FFJ, Scarano F (2008) Effect of predictor-corrector filtering on the stability and spatial resolution of iterative PIV interrogation. Exp Fluids 45(5):927-941. https://doi.org/10.1007/s0034 8-008-0511-7

Sciacchitano A, Wieneke B, Scarano F (2013) PIV uncertainty quantification by image matching. Meas Sci Technol 24(4):045302. https://doi.org/10.1088/0957-0233/24/4/045302

Seong JH, Song MS, Nunez D, Manera A, Kim ES (2019) Velocity refinement of PIV using global optical flow. Exp Fluids 60(11):174. https://doi.org/10.1007/s00348-019-2820-4

Stanislas M, Okamoto K, Kähler CJ, Westerweel J, Scarano F (2008) Main results of the third international PIV Challenge. Exp Fluids 45(1):27-71. https://doi.org/10.1007/s00348-008-0462-Z
Susset A, Most JM, Honore D (2006) A novel architecture for a superresolution PIV algorithm developed for the improvement of the resolution of large velocity gradient measurements. Exp Fluids 40(1):70-79. https://doi.org/10.1007/s00348-005-0047-z

Wang HP, Gao Q, Feng LH, Wei RJ, Wang JJ (2015) Proper orthogonal decomposition based outlier correction for PIV data. Exp Fluids 56(2):1-15. https://doi.org/10.1007/s00348-015-1894-x

Westerweel J, Geelhoed PF, Lindken R (2004) Single-pixel resolution ensemble correlation for micro-PIV applications. Exp Fluids 37:375-384

Wieneke B (2015) PIV uncertainty quantification from correlation statistics. Meas Sci Technol 26(7):074002. https://doi. org/10.1088/0957-0233/26/7/074002

Willert CE, Gharib M (1991) Digital particle image velocimetry. Exp Fluids 10:181-193

Xu Y, He GS, Kulkarni V, Wang JJ (2017) Experimental investigation of influence of Reynolds number on synthetic jet vortex rings impinging onto a solid wall. Exp Fluids 58(1):17. https://doi. org/10.1007/s00348-016-2287-5

Zhong QL, Yang H, Yin ZP (2017) An optical flow algorithm based on gradient constancy assumption for PIV image processing. Meas Sci Technol 28(5):055208. https://doi.org/10.1088/1361-6501/ aa6511

Zille P, Corpetti T, Shao L, Xu C (2016) Super-resolution of turbulent passive scalar images using data assimilation. Exp Fluids. https ://doi.org/10.1007/s00348-015-2104-6

Publisher's Note Springer Nature remains neutral with regard to jurisdictional claims in published maps and institutional affiliations. 